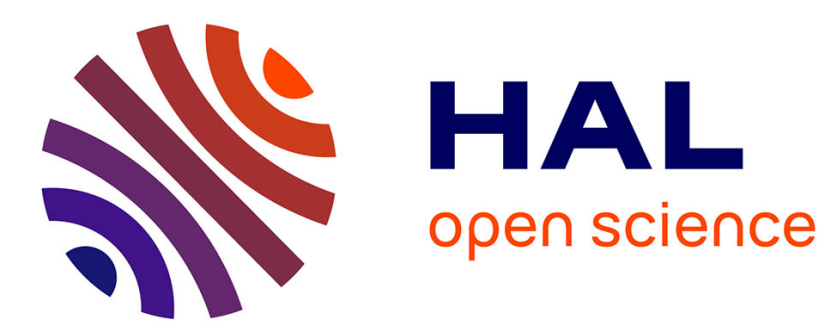

\title{
Experimental-Numerical Parametric Investigation of a Rocket Nozzle Secondary Injection Thrust Vectoring
}

\author{
V. Zmijanovic, L. Leger, E. Depussay, M. Sellam, Eric Depussay,
}

\section{To cite this version:}

V. Zmijanovic, L. Leger, E. Depussay, M. Sellam, Eric Depussay,. Experimental-Numerical Parametric Investigation of a Rocket Nozzle Secondary Injection Thrust Vectoring. Journal of Propulsion and Power, 2015, 32 (1), 10.2514/1.B35721 . hal-02398152

HAL Id: hal-02398152

https: / hal-univ-evry.archives-ouvertes.fr/hal-02398152

Submitted on 4 Jun 2021

HAL is a multi-disciplinary open access archive for the deposit and dissemination of scientific research documents, whether they are published or not. The documents may come from teaching and research institutions in France or abroad, or from public or private research centers.
L'archive ouverte pluridisciplinaire HAL, est destinée au dépôt et à la diffusion de documents scientifiques de niveau recherche, publiés ou non, émanant des établissements d'enseignement et de recherche français ou étrangers, des laboratoires publics ou privés. 


\title{
Experimental-Numerical Parametric Investigation of a Rocket Nozzle Secondary Injection Thrust Vectoring
}

\author{
Vladeta Zmijanovic, ${ }^{*}$ Luc Leger, $^{\dagger}$ and Eric Depussay ${ }^{\dagger}$ \\ Institute ICARE, Centre National de la Recherche Scientifique, 45071 Orléans, France \\ and \\ Mohamed Sellam ${ }^{\ddagger}$ and Amer Chpoun ${ }^{\S}$ \\ Université d'Evry Val d'Essonne, 91025 Evry, France
}

\begin{abstract}
Secondary transverse injection into the divergent section of an axisymmetric convergent-divergent propulsive nozzle is investigated for the fluidic thrust vectoring effects. Coupled experimental and numerical cold-flow investigation on the number of cases and aspects was conducted in the framework of a French microsatellite launcher program. Five experimental test nozzles were designed, built, and equipped with diagnostic tools. All experimental test models were supported by full three-dimensional numerical simulations and further investigated using the additional nozzle models, cases, and analysis parameters. Pertinent side force and pitch vector angle of 5-9 deg were achieved within the 5-8\% range of the secondary to primary mass-flow-rate ratio. Investigation aspects, categorized as the nozzle vectoring system geometrical characteristics, primary and secondary flow conditions, and gas intrinsic properties were found to dominantly affect the thrust vectoring capabilities. Some further improvements are suggested and achieved in the optimization of selected parameters.
\end{abstract}

\section{Nomenclature}

\begin{tabular}{|c|c|c|}
\hline$A$ & $=$ & cross-section area \\
\hline$C_{\mathrm{AF}}$ & $=$ & global force-amplification coefficient \\
\hline$C_{\mathrm{AI}}$ & $=$ & global specific impulse amplification coefficient \\
\hline$C_{\mathrm{AV}}$ & $=$ & vector amplification coefficient \\
\hline$C_{d}$ & $=$ & discharge coefficient \\
\hline$C_{\mathrm{pmax}}$ & $=$ & pressure coefficient corresponding to maximum angle \\
\hline$F_{a}$ & $=$ & axial thrust force \\
\hline$F_{j}$ & $=$ & secondary injection reactive force \\
\hline$F_{w}^{\prime}$ & $=$ & $\begin{array}{l}\text { force from main jet and second injection interaction } \\
\text { exerted on the nozzle wall }\end{array}$ \\
\hline$F_{x, y, z}$ & $=$ & force Cartesian components \\
\hline$F_{0}$ & $=$ & nonvectored nozzle thrust force \\
\hline$f_{m}$ & $=$ & secondary to primary mass-flow-rate ratio \\
\hline$h$ & $=$ & injectant plume Mach disk height \\
\hline$I_{\mathrm{sp}}$ & $=$ & specific impulse \\
\hline$M$ & $=$ & Mach number \\
\hline$M_{g}$ & $=$ & molecular weight of the gas species \\
\hline$\dot{m}_{j}$ & $=$ & secondary injection mass flow rate \\
\hline$\dot{m}_{0}$ & $=$ & primary mass flow rate \\
\hline$p_{a}$ & $=$ & ambient pressure \\
\hline$p_{j}$ & $=$ & secondary injection pressure \\
\hline$p_{p}$ & $=$ & plateau pressure \\
\hline$P_{0}$ & $=$ & main flow total pressure \\
\hline$q$ & $=$ & dynamic pressure \\
\hline$y+$ & $=$ & dimensionless wall distance \\
\hline$\gamma$ & $=$ & heat capacity ratio \\
\hline$\delta$ & $=$ & vector pitch angle \\
\hline$\theta$ & $=$ & central angle \\
\hline$\phi$ & $=$ & injection angle \\
\hline
\end{tabular}

\section{Introduction}

NVESTIGATION on the thrust vectoring effects of a transverse sonic injection into a divergent section of a supersonic rocket

*Research Associate, CNRS-Institute PPRIME, CEAT-Univ. Poitiers, 43 Route de l'aerodrome, 86036 Poitiers, France; vladeta.zmijanovic@ polytechnique.edu. Member AIAA.

Assistant Professor, CNRS-ICARE, 1C Av. de la recherche Scientifique.

${ }^{\ddagger}$ Assistant Professor, IUT Univ. d'Evry, Cours Monseigneur Romero.

${ }^{\S}$ Full Professor, IUT Univ. d'Evry, Cours Monseigneur Romero. nozzle was conducted in the framework of the small satellite space launcher program "Perseus" [1] of the Centre National d'Etudes Spatiales (CNES) French space agency. This study investigates the possibilities and prospective implementation of a secondary injection thrust vector control (SITVC) system as a part of the PERSEUS macroproject aimed at new and immature technologies for a microsatellite space launcher. A possible suppression of complexity and weight by elimination of heavy and robust mechanical actuators and a further increase in dynamic responsiveness highlight the fluidic thrust vectoring method as a desirable alternative, which is especially attractive for a small and compact launcher propulsive system.

Following the number of investigations that were based on the empirical, experimental, and analytical approaches to the problem in the 1960s and 1970s, as well as numerical studies on supersonic crossflows in 1990s and early 2000s, the current study pays further attention to the possible operational regimes and implementation aspects of the SITVC system.

Among several modes of fluidic thrust vectoring, such as counter [2] and coflow methods [3] or skewing of a sonic line [4], SITVC was selected as a straightforward method, with its advantage in simplicity of implementation and installation.

In general, secondary injection in a supersonic nozzle acts as an obstacle and source of momentum change to the oncoming supersonic flow. As a response to the blockage in supersonic expanding flow, a strong bow shock is formed inside the main flow, imposing an adverse pressure gradient (APG) upstream of the injection, as in $[5,6]$. This causes an asymmetric upstream separation of the portion of the main flow from the nozzle wall that generates an imbalance in the pressure net force. The main nozzle supersonic flow then deflects through the leading shock, and this shock-flow interaction results in generation of a side force component, which is imposed on the separated flow section of the nozzle wall. The second component of the side force can be identified as a natural reactive force of secondary transverse injection, as denoted in Fig. 1. Zukoski and Spaid [7] and Spaid and Zukoski [8] found that secondary slot injection at the flat plate, illustrated in Fig. 2, acts on the oncoming supersonic flow in a similar manner as the solid blunt body. Their blunt-body model proved to be satisfactorily accurate for the moderate secondary to primary mass-flow-rate ratios, as reported in [9-11], and was comparable with the empirical models given in [12-14] and others. 


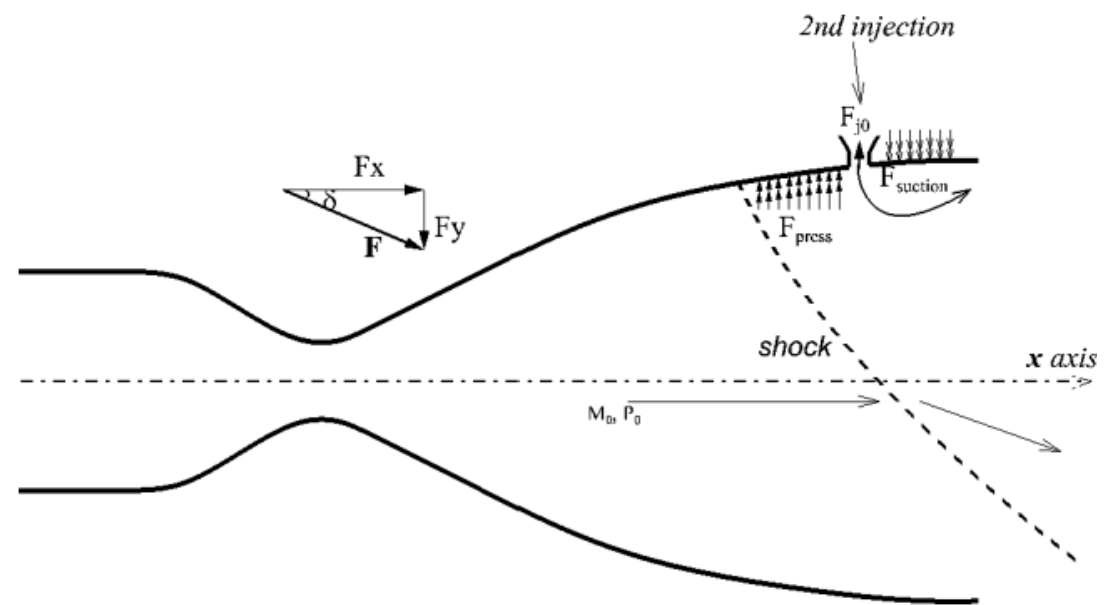

Fig. 1 Scheme of the CD nozzle shock vector control.

The fundamental three-dimensional (3-D) case, that is represented by a circular sonic injection into the supersonic crossflow on a flat plate features the flow effects in all three spatial directions, as depicted in Fig. 3. The upstream separation distance according to $[5,15]$ is defined by the nature of upstream flow (laminar-turbulent) and by the magnitude of the adverse pressure gradient $[16,17]$. At the detachment incipient point, a weaker separation shock emerges due to an adverse pressure and, further downstream, it interacts through the compression fan and merges into a strong bow shock. Inside the separation region, a recirculation zone is formed involving two counter-rotating vortices named, respectively, the primary upstream vortex (PUV) and the secondary upstream vortex (SUV). In the 3-D case $[6,18]$, a horseshoe-shaped vortex is formed that wraps around the injectant plume, with the 3 -D bow shock region surrounding this interaction zone. The injectant is underexpanded, and it enters the main flow with a Prandtl-Meyer expansion fan that is formed at the injection orifice. The barrel shock surrounds its recompression with the Mach disk at the end of the first recompression sequence. High pressure imposed on the secondary plume windward side and low pressure on its leeward side cause the inward folding of the secondary jet with accompanying interior shock reflections. After this first sequence, trailing kidney-shaped vortices interact with the horseshoe vortex, generating an effective mixing layer, as pointed in [19]. Downstream of the injection port, wake vortex shedding takes place, which involves a primary downstream vortex (PDV), eventual secondary vortex and flow reattachment with accompanying recompression shock.

Compared to the fundamental cases of slot and circular sonic injections at the flat plate, the secondary injection inside a rocket nozzle is distinguished by the strong wall-bounded supersonic crossflow character, as was also reported in [20-24]. The internal

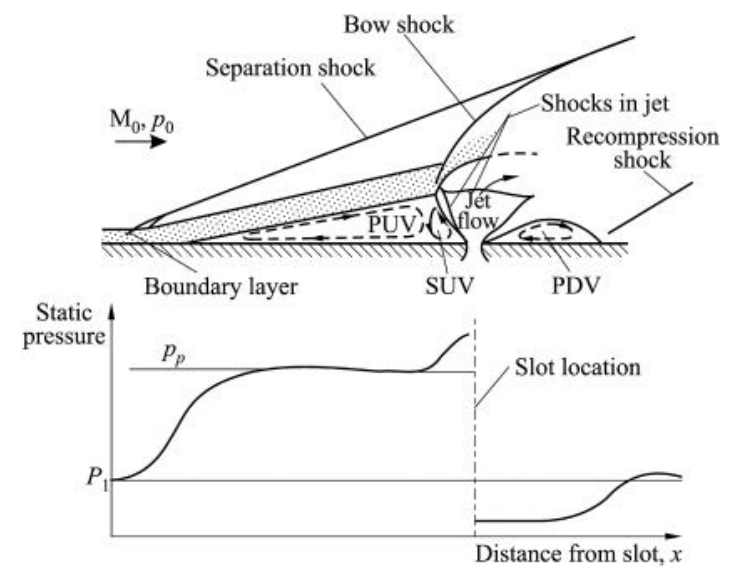

Fig. 2 Schematic of 2-D transverse slot injection model and wallpressure distribution (reprinted from [7] with permission from the AIAA). effects of the lateral sides of the wall and the possible main shock reflection further affect the complex interaction crossflow field. In the case of secondary injection inside the nozzle, it is of substantial interest to determine the separation zone in the nozzle, the pressure distribution, and the eventual effects of the shock interaction and shock-wall reflection.

To quickly assess the basic effects and prepare experimental investigation, an analytical model was adopted. This model was based on the modified Zukoski-Spaid blunt-body approximation [7], with the addition of several nozzle flow separation criteria, as previously described in $[25,26]$. Starting from the momentum balance relation on the control volume in Fig. 4, the model solves equations for penetration height and upstream separation distance. Pursuant to the two-dimensional (2-D) scheme in Fig. 4, in the 3-D domain, secondary injection is represented as a quarter-sphere defined by the radius $h$, which is followed by the half-open aft cylinder. The balance relation implies that the net pressure force, or drag, acting on the control volume boundary surface is equal to the momentum of a fluid leaving the domain. According to [26], with a no-mass exchange assumption, it can be written as

$$
\int_{A B}-p \boldsymbol{x} \mathrm{d} A+\int_{C D}-p \boldsymbol{x} \mathrm{d} A=\int \rho \boldsymbol{U} \boldsymbol{U} \mathrm{d} A
$$

The net force acting on the control volume in the $x$ direction may be decomposed on the pressure forces acting on the upwind section of the interface and the momentum force at the exit of the control volume:

$$
\sum F_{x}=\int \rho u^{2} \mathrm{~d} s=\int_{0}^{\pi / 2} p \cos \theta \mathrm{d} A-p_{i} \frac{\pi}{2} h^{2}
$$

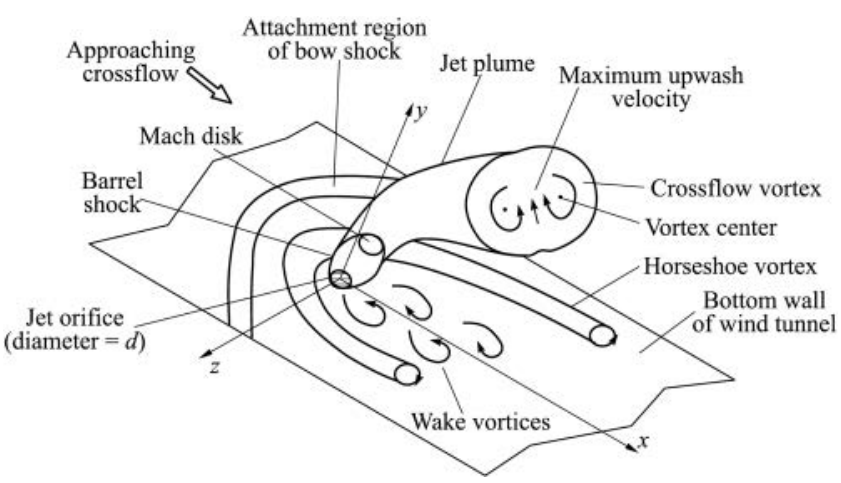

Fig. 3 Schematic of 3-D transverse circular injection on the flat plate (reprinted from [18] with permission from the AIAA). 


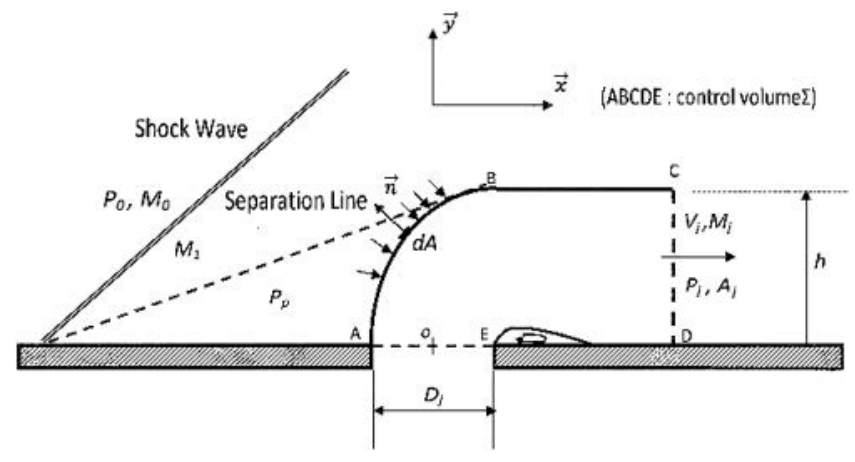

Fig. 4 Scheme of control volume zone.

Solved for the radius $h$, this integration yields

$$
\sum F_{x}=\pi h\left(\frac{p_{0}-p_{i}}{2}+\frac{1}{4} q_{0} C_{\mathrm{pmax}}\right)
$$

where $C_{\mathrm{pmax}}$ represents the pressure coefficient corresponding to the maximum angle $\theta$ of $\pi / 2$, between the incident pressure force direction and the normal to the wall. This relation is complemented with the one-dimensional (1-D) isentropic momentum relation of a sonically injected secondary flow:

$$
\int \rho v^{2} \mathrm{~d} s=\dot{m}_{j} V_{j}
$$

After isentropic transformations, this equation yields

$$
\begin{aligned}
\dot{m}_{j} V_{j} & =2 C_{d} A_{j} \gamma_{j} P_{0 j}\left(\frac{2}{\gamma_{j}-1}\right)^{1 / \gamma-1} \\
\cdot & {\left[\frac{1}{\gamma_{j}^{2}-1}\left(1-\left(\frac{p_{j}}{P_{0 j}}\right)^{\gamma_{j}-1 / \gamma_{j}}\right)\right]^{1 / 2} }
\end{aligned}
$$

where $C_{d}$ and $A_{j}$ represent the discharge coefficient and area of the circular injector surface, respectively. Substitution of the corresponding terms in Eq. (4) leads to an expression that evaluates the penetration height:

$$
h_{j}=D_{j} \sqrt{\frac{C_{d} \gamma_{j} P_{0 j}\left(2 / \gamma_{j}-1\right)^{1 / \gamma-1} \cdot\left[1 / \gamma_{j}^{2}-1\left(1-\left(p_{j} / P_{0 j}\right)^{\gamma_{j}-1 / \gamma_{j}}\right)\right]^{\frac{1}{2}}}{\left(p_{0}-p_{i}\right)+q_{0} C_{\mathrm{pmax}} / 2}}
$$

The penetration height equation is coupled with the selected separation criteria in order to delineate the nozzle flow separation region. Empirical free-shock-separation (FSS) criteria, such as the one of Schilling [27], are considered for evaluation of separation pressure:

$$
\frac{p_{\text {sep }}}{P_{0}}=k_{1}\left(\frac{P_{0}}{p_{a}}\right)^{k_{2}}
$$

where $k_{1}=0.541$ and $k_{2}=-0.136$ denote the Shilling empirical coefficients for convergent-divergent (CD) nozzle cold flow. As reported in [25], the preceding relations were paired with the FSS criterion of Green [28], whereas the separation line along the nozzle wall, illustrated in Fig. 5, was evaluated according to the procedure of detached shock waves forming around the spherical and cylindrical nosed bodies, as in [29].

To quantify the observed effects a certain metrics is proposed. The vectoring or pitching angle $\delta$ is defined as the arctangent of the ratio between the pressure forces and momentum fluxes in the $y$ direction and the ones in the $x$ direction:

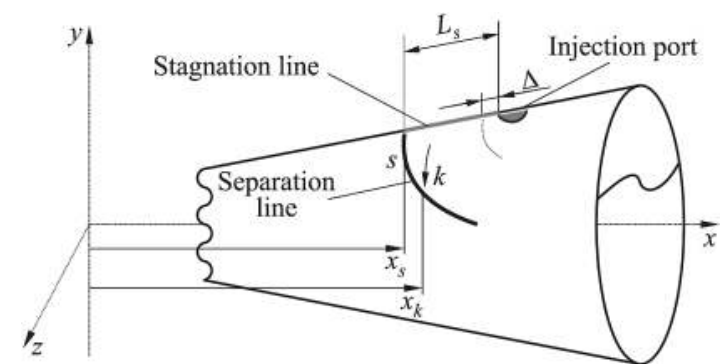

Fig. 5 Scheme of separation line alongside nozzle wall.

$$
\delta=\arctan \left[\frac{F_{y}}{F_{x}}\right]=\tan ^{-1}\left[\frac{\sum f_{Y}+\sum(\dot{m} u)_{y}}{\sum f_{X}+\sum(\dot{m} u)_{x}}\right]
$$

Factors that influence the performances and losses of vectored and nonvectored nozzles [12,21,30,31] were of particular interest in the parametric analysis of the SITVC nozzle. To correctly evaluate the contributions of secondary injection to the nozzle performance, coefficients that represent vectoring $C_{A V}$, force $C_{\mathrm{AF}}$, and global specific impulse $C_{\mathrm{AI}}$ amplification are considered together with the corresponding pitch vector angle, in a manner similar to the procedure previously proposed in [32]:

$$
C_{\mathrm{AV}}=\frac{\arctan \left(F_{y} / F_{x}\right)}{\left(\dot{m}_{j} / \dot{m}_{0}\right) \cdot 100 \mathrm{deg}}
$$

$$
\begin{gathered}
C_{\mathrm{AI}}=\frac{I_{\mathrm{sp}}^{*}}{I_{\mathrm{sp} 0}}=\frac{\sqrt{F_{x}^{2}+F_{y}^{2}} / \dot{m}_{0}+\dot{m}_{j}}{F_{0} / \dot{m}_{0}} \\
C_{\mathrm{AF}}=\frac{\sqrt{F_{x}^{2}+F_{y}^{2}}}{F_{0}}
\end{gathered}
$$

\section{Experimental Setup and Test Models}

Cold-flow experiments in this study were conducted in the super/ hypersonic wind-tunnel facility of the Centre National de la Recherche Scientifique, Institut de Combustion Aérothermique Réactivité et Environnement using the five main and three secondary injection chamber-nozzle configurations.

Wind-tunnel EDITH, previously SH2 [14], of the Aerothermic Laboratory, was newly equipped, configured, and set by the authors of this study. Dry and oil-free air stored under 300 bar in a 320 liter reservoir was pressure regulated before the settling chamber and then expanded through the rocket nozzle into the vacuum test section of

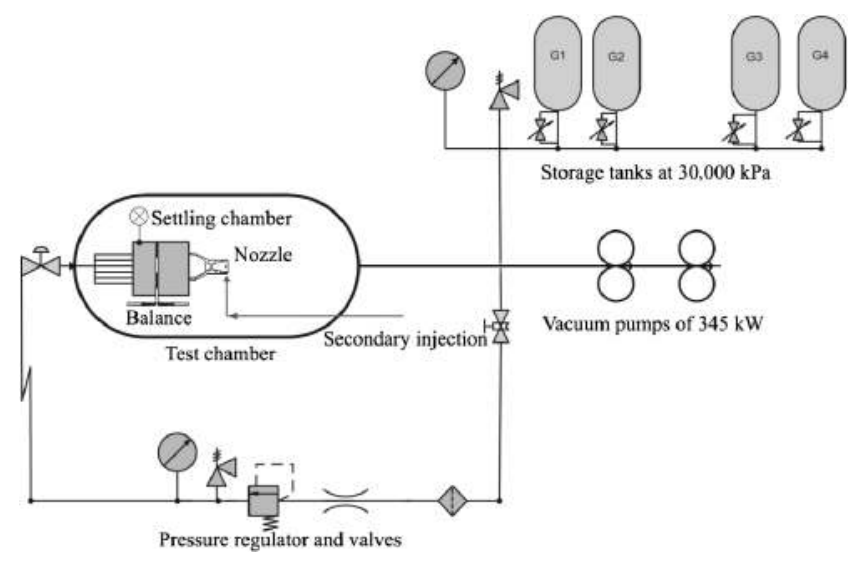

Fig. 6 Scheme of the wind-tunnel EDITH operation cycle. 
Table 1 EDITH setup aerodynamic conditions

\begin{tabular}{lc}
\hline Stagnation conditions & Ambient and freestream conditions \\
\hline$P_{0}=300 \mathrm{kPa}$ & $p_{a}=8 \mathrm{kPa}$ \\
$T_{0}=260 / 243 \mathrm{~K}$ & $T_{a}=290 \mathrm{~K}$ \\
$\rho_{0}=4.26 \mathrm{~kg} / \mathrm{m}^{3}$ & $\rho_{a}=0.098 \mathrm{~kg} / \mathrm{m}^{3}$ \\
$N P R_{D}=37$ & $U_{e}=574.73 \mathrm{~m} / \mathrm{s}$ \\
$A_{\mathrm{th}} / A_{e}=0.236$ & $\rho_{e}=0.3142 \mathrm{~kg} / \mathrm{m}^{3}$ \\
$\dot{m}_{0}=236 \mathrm{~g} / \mathrm{s}$ & $M_{e}=3$ \\
& $R_{e} / m=2.7729 \cdot 10^{7} / \mathrm{m}$ \\
\hline
\end{tabular}

the wind tunnel, as illustrated in Fig. 6. General characteristics of this experimental setup are given in Table 1 .

Several qualitative and quantitative diagnostic techniques were applied to the experimental test models in this study. Flowfield visualization mainly relied on the Toepler's $Z$ schlieren that was capturing density gradient flow effects at the nozzle exit section, as described in [25]. Quantitative measurements of forces, pressures, and temperatures were performed using the designed force balance, Kulite XCQ-062 parietal pressure sensors, a Scanivalve ZOC-22B pressure scanner, and thermocouples at each section of the flow.

The constructed force balance consisted of HBM S2M S-shaped strain-gauge force transducers and three moving frames connected via frictionless slide bearings to the three-axes moving test model and attached to the test-section construction support. Two force transducers of $200 \mathrm{~N}$ range were mounted on the vertical $y$ axis to capture the vectoring side force: one transducer on the $x$ axis measuring the thrust force, and small $20 \mathrm{~N}$ range transducers in the lateral $z$ direction to monitor eventual asymmetric loads. The transducer signal was amplified to the $0-10 \mathrm{~V}$ range via an HBM RM4220 before fast acquisition by National Instruments SCXI1140 cards.

All components were calibrated using a series of small weights and then recalibrated with a fully mounted test model. The obtained result data were accompanied with the standard deviation and total error margin.

Parietal pressures were measured via fast pressure probes and pressure taps that were distributed along the nozzle meridians, as illustrated in Fig. 7. Eleven Kulite XCQ-062 probes were placed on the injection meridian at the symmetry plane and at characteristic lateral positions in order to delineate the separation region and to monitor its propagation. The other 32 pressure taps of $1 \mathrm{~mm}$ diameter were sorted along the meridians defined at 13, 20, 30, 36, 45, 60, 75, and $90 \mathrm{deg}$, as well as on the side opposite the injection. Kulite pressure signals were acquired with a $8 \mathrm{kHz}$ sampling rate, whereas a Scanivalve ZOC-22B pressure scanner operated at $0.5 \mathrm{kHz}$ per channel.

All employed diagnostic tools were calibrated and the error analyzed before reporting; calibration and error estimation were reported in more detail in appendix B of [33]. The maximal
Table 2 Experimental test nozzle design parameters

\begin{tabular}{lccccccc}
\hline Nozzle & $R_{\mathrm{th}}, \mathrm{mm}$ & $A_{e} / A_{\mathrm{th}}$ & $M_{e}$ & $\mathrm{NPR} D i$ & $l_{n}, \mathrm{~mm}$ & $x_{i} / l_{n}$ & $A_{t j}, \mathrm{~mm}^{2}$ \\
\hline Conical C-i0.7 & 9.72 & 4.234 & 3. & 37 & 100 & 0.7 & 32.17 \\
Conical C-i0.9 & 9.72 & 4.234 & 3 & 37 & 100 & 0.9 & 28.274 \\
TIC-i0.88 & 10 & 4.87 & 3.03 & 37.5 & 68 & 0.88 & 26.42 \\
\hline
\end{tabular}

TIC, truncated ideal contour.

cumulated uncertainty of $\pm 0.5 \%$ was considered for Kulite XCQ0.62 pressure probes and presented via error bars in all experimental pressure figures in the Results Discussion section (Sec. IV). This error estimation combined nonlinearity, hysteresis, and repeatability of the pressure probes, similar to [34]. Error estimation of the force balance system considered calibration of each force transducer, as well the cumulative calibration and cumulative error estimation of a mounted system under static and dynamic series of small loads, as reported in [33]. The standard deviation and estimated uncertainty for the vertical axis, where side force was measured, was up to $\pm 2 \%$ of the measured force; whereas in the $x$ direction, uncertainty was estimated up to $\pm 0.5 \%$. In the result graphs and tables, the $8 \mathrm{kHz}$ sampled force data were averaged on 2000-sample chunks and presented with the corresponding uncertainty estimations.

After preliminary tests with a conical-shaped supersonic nozzle, reported in [25], the main investigation was concentrated on a truncated ideal contour (TIC) nozzle intended for the CNES microsatellite launcher. TIC nozzle models were adapted for Mach 3 with a designed large and uniform subsonic to supersonic throat curvature radius $\left(R_{c}=2 r_{\text {th }}\right)$. The axisymmetric ideal nozzle contour was calculated using the method of characteristics by the procedure given in [35]. As this calculation produced an inviscid result, the resulting profile was corrected for the low wall-evolving boundarylayer thickness. The basic design parameters of test nozzles are given in Table 2. Several injection positions and one inclination angle were experimentally investigated on a conical contour with a constant slope ratio; whereas secondary injection types, modes, and (finally) flow regimes were tested on TIC nozzle test models. Secondary flow was supplied from the secondary settling chamber into the secondary injection convergent nozzle and smoothly injected at the divergent section of the main nozzle through the sonic throat of $28 \mathrm{~mm}^{2}$. Two injection convergent nozzle types were used: axisymmetric with a circular profile, and slot injection with a rounded rectangular profile. The aforementioned throat size of the injection nozzle provided a mass-flow-rate ratio of $8 \%, f_{m}=m_{j} / m_{0}=0.08$ under the secondary-to-primary pressure ratio (SPR) condition equal to one.

The same test nozzles were designed, meshed, and used in the numerical simulations of this investigation. After confronting and coupling experimental and numerical data, additional numerical tests were conducted for higher-Mach-number nozzles and different contours, such as Rao's thrust-optimized [36] and adverse-pressuregradient [37] nozzle types.

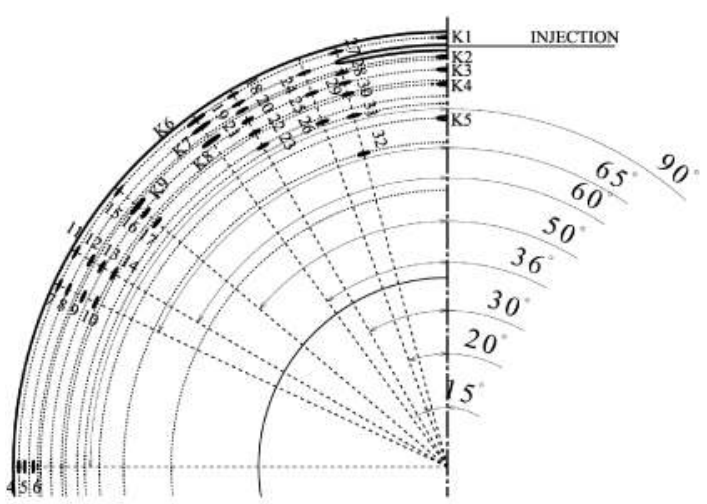

a) CAD scheme of meridional parietal pressure probe distribution

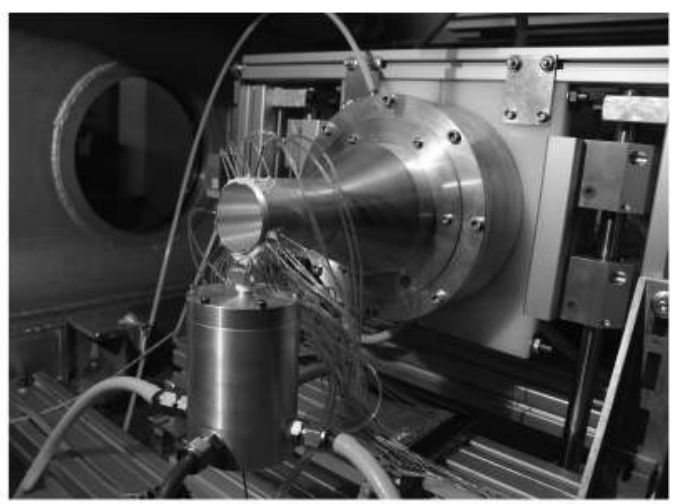

b) Equipped and mounted test nozzle

Fig. 7 Experimental test bench. 


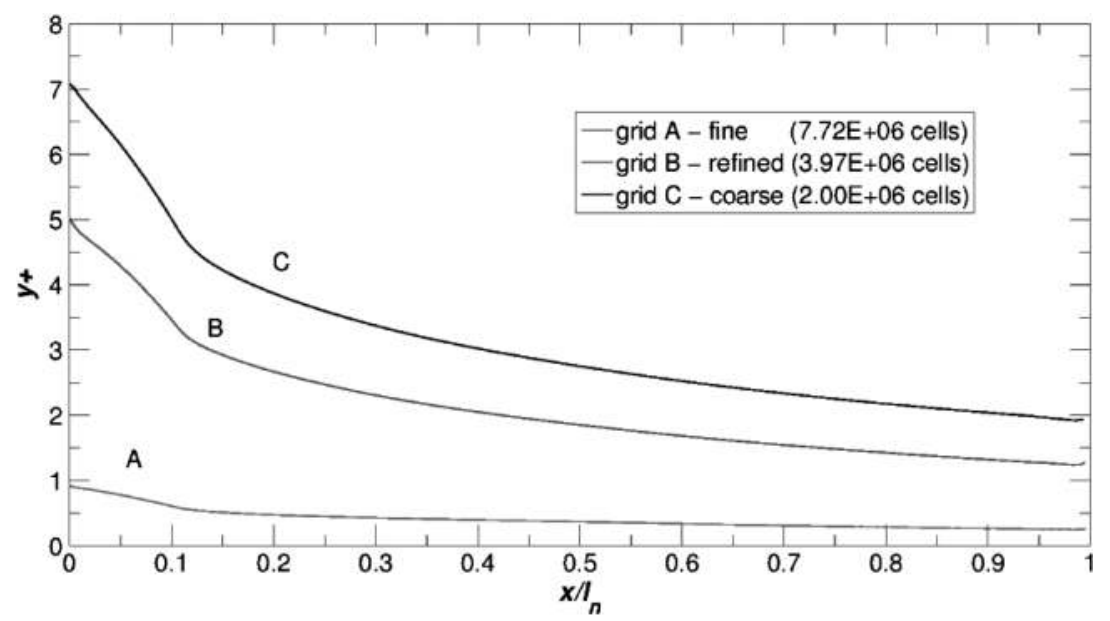

Fig. $8 y+$ of 3-D grid for three mesh quality sequences.

\section{Numerical Test Environment}

A computational fluid dynamics (CFD) approach has been considered in this investigation as a coupled method with the experiments in order to provide a reliable comparison and give a more detailed insight analysis of the experimentally observed phenomena. Mass-averaged Navier-Stokes equations were solved using the finite volume CFD solver CPS_C (Code pour la Propulsion Spatiale Cryo) [38].

CNES and Bertin Technologies's CPS_C is a three-dimensional CFD code designated for compressible multispecies reacting flows with fully accounted viscous effects on an unstructured 3-D computational grid. Favre-averaged Navier-Stokes equations are solved using the explicit schemes of a modified second-order RungeKutta, which can be of an order up to four in time and three in space. Transport models use a multichemical species mixing law of a convection/diffusion fluxes type based on Fick's law. The fluxes in this numerical work were computed at the cell interfaces with Harten-Lax-van Leer contact scheme by Toro [38,39].

A standard two-equation turbulence Jones-Launder $k-\varepsilon$ model was implemented and coupled to the CPS solver. As depicted in Fig. 8, the $y+$ value in the nozzle computation domain was below one for the reported fine grid; therefore, no wall function was applied in this case. In the domains with a coarser grid, as in the external region close to the far-field and outlet boundaries, the wall function was switched on for $y+$ values of 11 and above. The adiabatic wall function was coupled to the turbulence model via the modified logarithmic law of van Driest. Compared to [40], the implemented $k-\varepsilon$ turbulence model in CPS code was adequate and performed well with the selected test models of the current study, following the good performance reported in [41]. The fine-grid $(A)$ quality sequence that consisted of 7.7 million elements was selected among the three other mesh quality sequences, and it is presented in the results of this study.

The numerical grid was based on mapped hexagonal elements using a rounded hexagonal-core $\mathrm{O}$ grid in the reference cross sections and with sweeping in between, as illustrated in Fig. 9. The mesh was clustered at the primary and secondary throat regions and toward the injection zone, whereas it was stretched in the exterior zone toward the outlets.

The computational domain consisted of a convergent nozzle zone, a throat, and a divergent nozzle zone. In the divergent section, special attention was paid to the injection nozzle region, whereas the exterior domain served as a smooth introduction to the outlet boundaries. This exterior domain, as depicted in Fig. 9b, was constructed as a cylindrical section of 14 nozzle exit diameters in the radial direction, 20 in the axial downstream direction, and 7 in the upstream direction, preventing any numerical outlet convective outflow effect on the nozzle exit region.

The species physical properties were modeled according to a thermally perfect gas assumption. Specific heat capacities were defined as a seventh-order polynomial temperature function derived from the Chemkin-II thermodynamic data. Accordingly, the laminar viscosity and conductivity were analytically formulated as fourthdegree temperature-dependent polynomial functions.

In evaluation of the flow effects at the nozzle wall, the compressible form of the pressure coefficient and skin friction was considered:

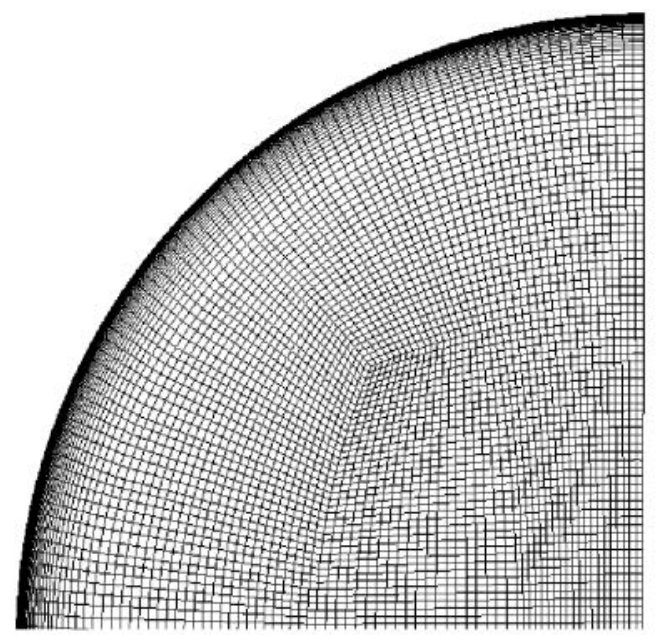

a) Nozzle cross-sectional mesh

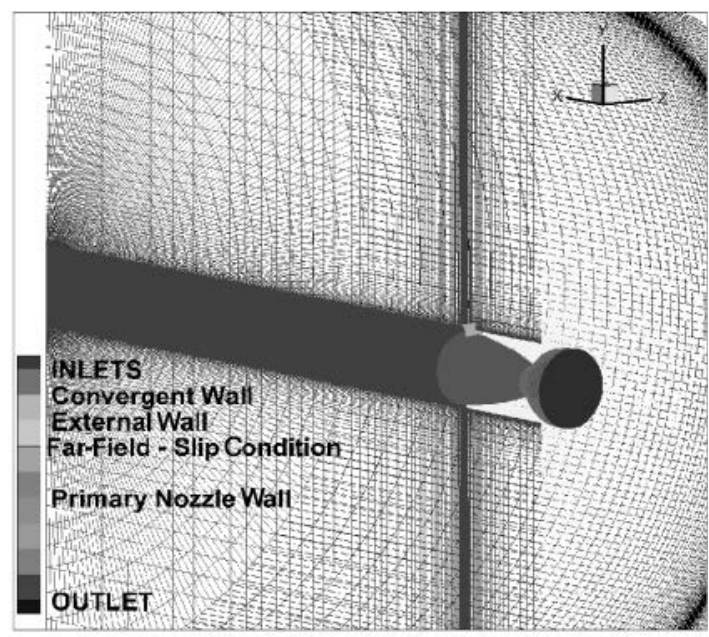

b) 3-D isoscheme of computational model

Fig. 9 Computational domain and boundaries. 


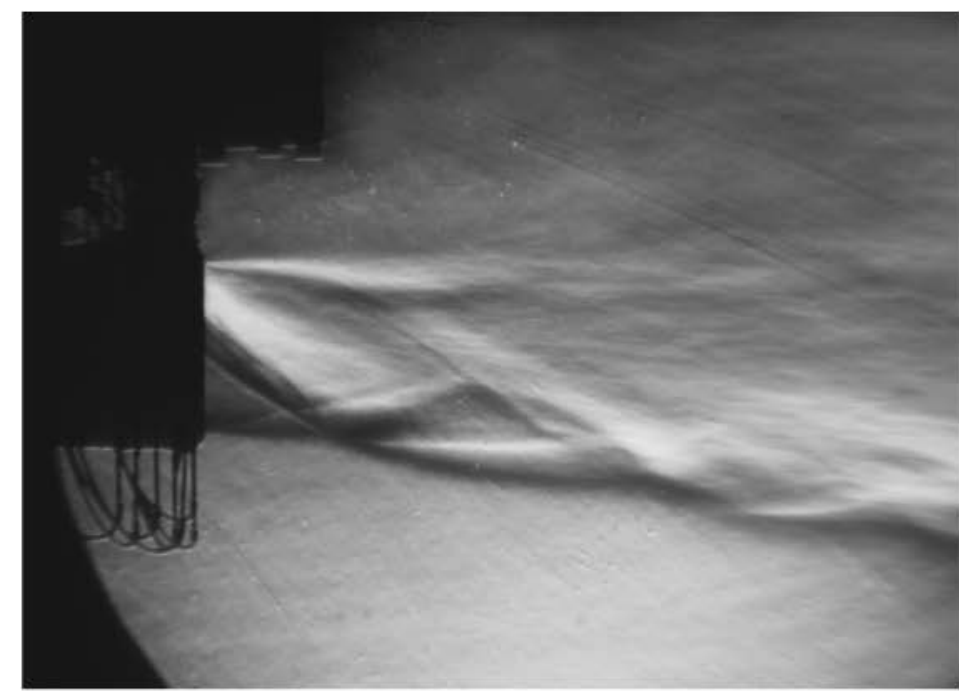

a) Experimental Z-schlieren photograph

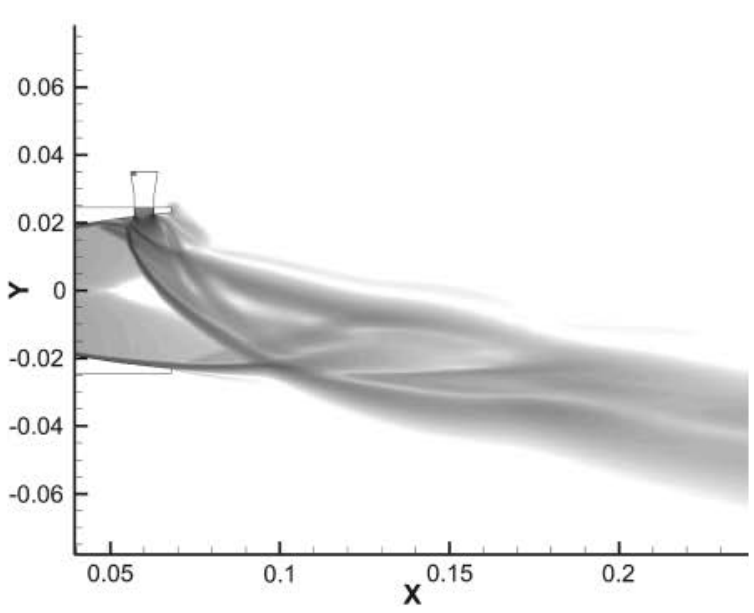

b) Numerical schlieren

Fig. $10 x y$ view of TIC nozzle with $x_{j} / l_{n}=0.88$ injection at $\mathrm{NPR}=37.5$, SPR $=1$, and $\delta=6.8 \mathrm{deg}$.

$$
c_{P}=\frac{2}{\gamma \cdot M_{\infty}^{2}}\left(\frac{p}{p_{\infty}}-1\right) \mid C_{f}=\frac{\tau_{w}}{P_{0}}
$$

where $\tau_{w}$ denotes the wall-shear stress obtained from the velocity in the first mesh cell close to wall and from the geometric transformation of that cell's coordinates. Alternatively, in the presented profiles, the pressures were nondimensionalized by the magnitude of total pressure.

\section{Results Discussion}

The experimental and numerical results data of the investigated aspects are analyzed and presented in an integrated manner. The results discussion is mainly concentrated on the crossflow properties, the primary and secondary gas flow effects, and the geometrical parameters of the SITVC nozzle [42].

\section{A. Secondary Injection Nozzle Interaction Crossflow Field}

By consulting the schlieren pictures in Figs. 10 and 11 of the truncated ideal contour nozzle model, some basic features of the secondary injection crossflow field can be observed. Namely, in this case, a secondary injection is introduced to the main flow at the divergent nozzle section closer to the exit. Therefore, the separation and bow shock leave the nozzle without interacting with the nozzle wall. In this shock-reflection-free case, the upstream detached boundary layer is accompanied by the weaker separation shock that, downstream, closer to the injection, transforms via compression waves into the strong bow shock that is formed in front of the injection plume. The top view in Fig. 11 depicts the lateral propagation of the separation and the formation of horseshoe-shaped vortex region that wraps around the secondary injection port and its plume. We can consider that there is no mixing occurring between the injectant and the main flow inside the nozzle for the given case and that a strong shock interface is formed between them.

The evaluated Mach number plots from the numerical solution in Fig. 12 point out this separation sequence evolution. Analogous to [43], we can delineate several distinctive regions in the nozzle flow at the exit cross section in Fig. 12b. These regions are denoted here as the low-pressure region behind the injection port (region A); the secondary plume Mach disk (region B); the interface region encircled by the Mach disk, bow shock, and horseshoe vortex (region C); the undisturbed main flow (regions $\mathrm{F}$ and $\mathrm{D}$ ); and the undisturbed main nozzle boundary layer (region $\mathrm{E}$ ).

In general, two major vector components of the global side force are identified: the wall side force, which is a result of the crossflow interaction; and a reactive force of the secondary transverse injection. The crossflow interaction force component is governed by an adverse

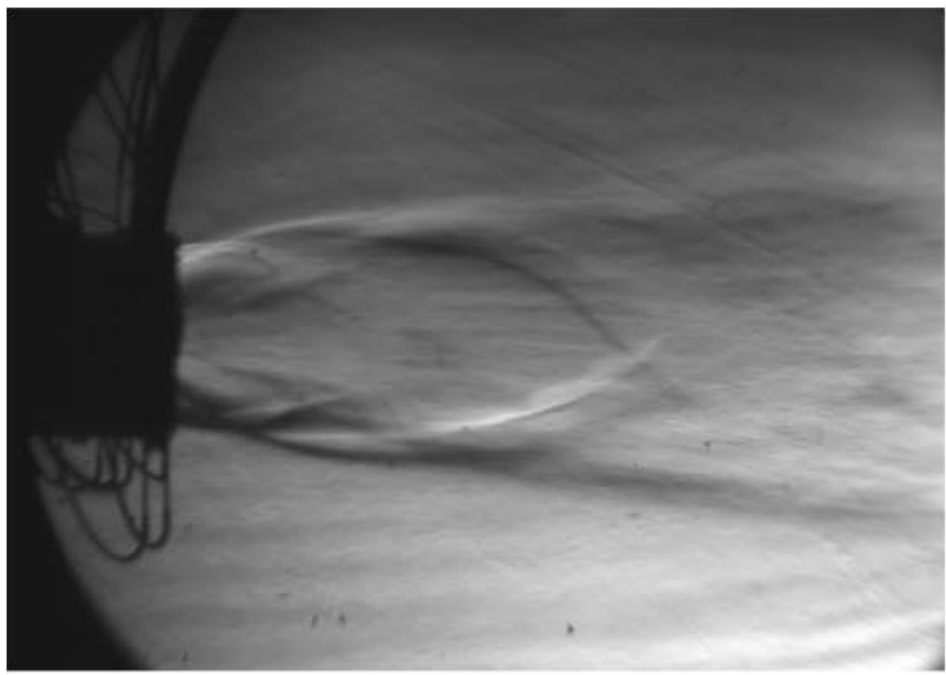

a) Experimental Z-schlieren photograph

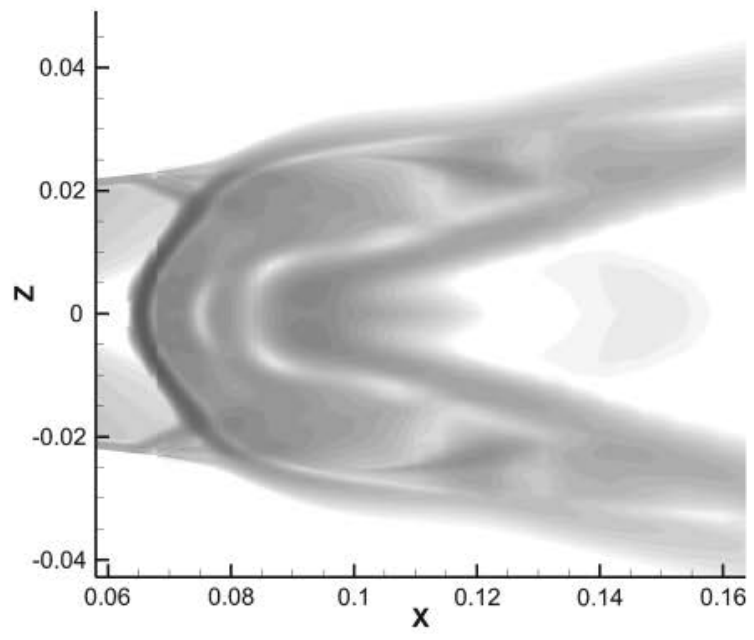

b) Numerical schlieren

Fig. $11 x z$ view of TIC nozzle with $x_{j} / l_{n}=0.88$ injection at NPR $=37.5, \mathrm{SPR}=1$, and $\delta=6.8 \mathrm{deg}$. 


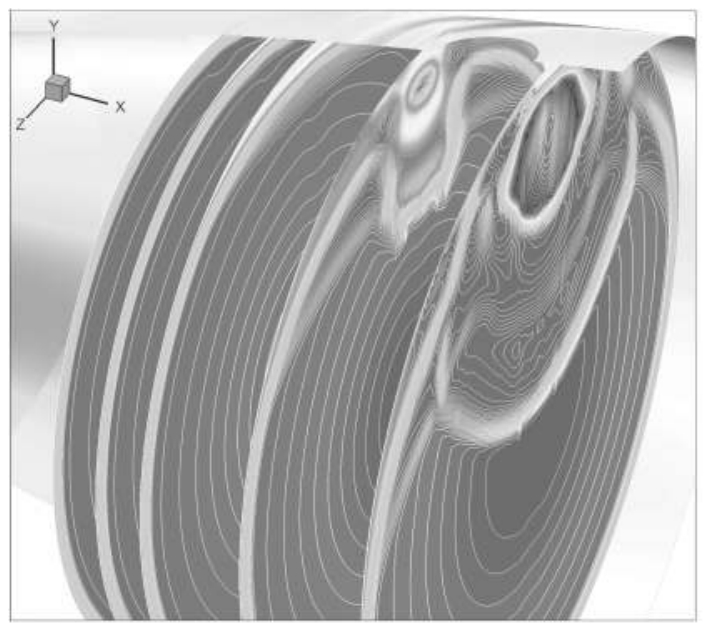

a) $y z$ slices toward the nozzle exit

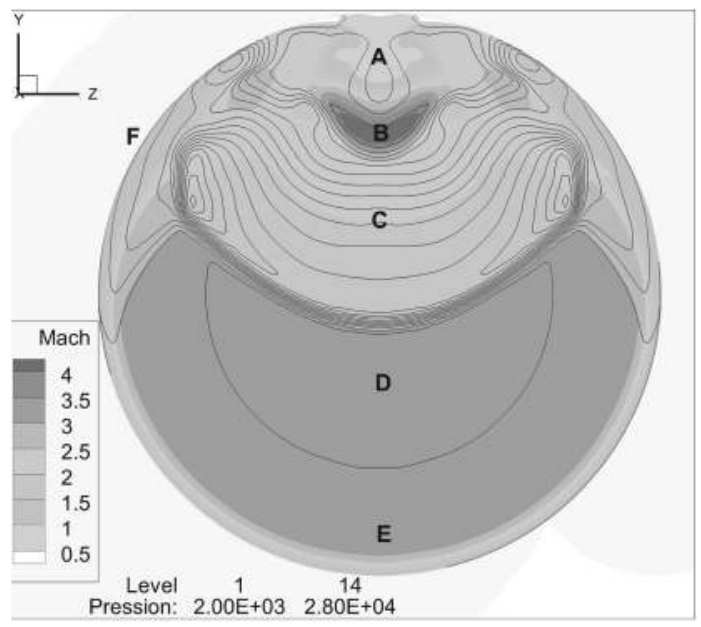

b) Exit plane of the TIC nozzle

Fig. 12 SITVC $x_{j} / l_{n}=0.88$ nozzle Mach number plot with pressure level lines.

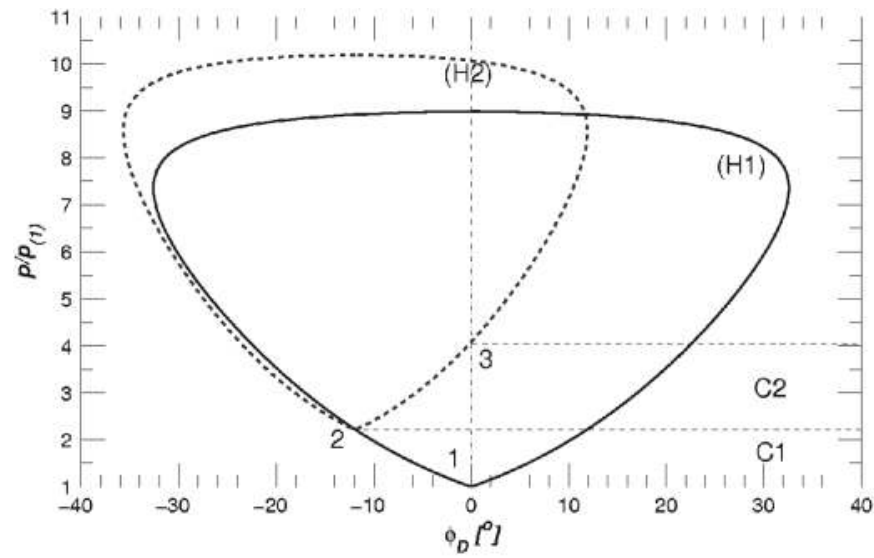

a) Regular reflection, $\varphi_{D}=11.5^{\circ}$

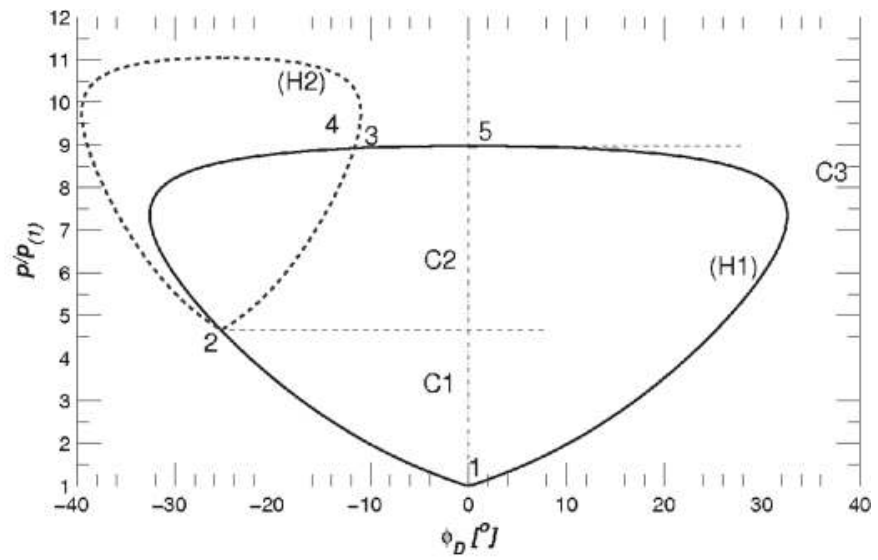

b) Mach phenomenon, $\varphi_{D}=25^{\circ}$

Fig. 13 Combined shock polar diagrams for incident oblique shock wave at $M 1=2.6$ in the polar plane.

pressure gradient and controlled via either changing the primary or secondary flow properties or controlling the geometrical parameters. The second force component is influenced by the nature of the secondary injection, which is primarily affected by the total pressure and temperature ratio of the injected gas and its physical properties. In Fig. 13a, the shock polar (H1 heart curve) represents incident planar shock wave $\mathrm{C} 1$ in an approximated $M=2.6$ uniform 1-D flowfield. For the initially evaluated negative deflection $-\Delta \varphi_{D}=11.5 \mathrm{deg}$, it is possible to represent state 2 with reflected $\mathrm{C} 2$ shock and corresponding heart curve $\mathrm{H} 2$. In this idealized case, $\mathrm{H} 2$ intersects the
$\varphi_{D}=0$ axis at point 3 , illustrating a nonrealistic regular reflection case that never happens for an axisymmetric nozzle. With an increase of deflection angle, $\mathrm{C} 1$ corresponds to the stronger shock solutions; thus, $\mathrm{H} 2$ does not intersect the 0 axis. Hence, the reflection point cannot exist on the wall any longer. To satisfy the slip boundary condition, we can define a triple point $T$, which exists between points 3 and 4 in Fig. 13b, along with the Mach disk (C3).

Analyzing the evaluated wall-pressure profiles in the symmetry plane, we can define several characteristic regions, as depicted in Fig. 14. After the incipient separation point, there is a steep pressure

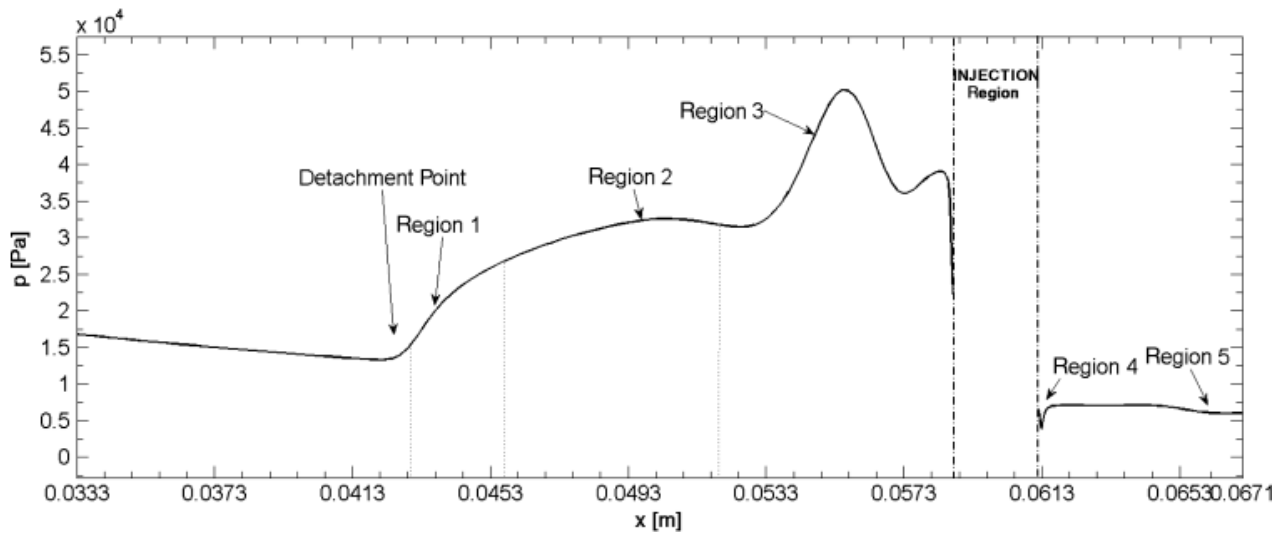

Fig. 14 Wall-pressure profiles in symmetry plane of TIC nozzle with injection at $x_{j} / l_{n}=0.88$. 
Table 3 Experimental statistical average data for range of second injection pressures in TIC nozzle at $x_{j} / l_{n}=0.88$

\begin{tabular}{lcrrr}
\hline$S P R=P_{j 0} / P_{0}$ & $\dot{m}_{j} / \dot{m}_{0}$ & \multicolumn{1}{c}{$F_{y}, \mathrm{~N}$} & \multicolumn{1}{c}{$F_{x}, \mathrm{~N}$} & $\delta, \mathrm{deg}$ \\
\hline$S P R=0.5$ & 0.038 & $7.49 \pm 2 \%$ & $135 \pm 0.5 \%$ & $3.18 \pm 4 \%$ \\
$S P R=0.667$ & 0.051 & $10.08 \pm 2 \%$ & $134.9 \pm 0.5 \%$ & $4.58 \pm 4 \%$ \\
$S P R=0.833$ & 0.063 & $12.37 \pm 2 \%$ & $136.06 \pm 0.5 \%$ & $5.20 \pm 4 \%$ \\
$S P R=1.00$ & 0.076 & $16.17 \pm 2 \%$ & $136.1 \pm 0.5 \%$ & $6.78 \pm 4 \%$ \\
$S P R=1.167$ & 0.089 & $18.9 \pm 2 \%$ & $137.7 \pm 0.5 \%$ & $7.82 \pm 4 \%$ \\
\hline
\end{tabular}

Table 4 Numerical averaged data for range of second injection rates in TIC nozzle $x_{j} / l_{n}=0.88^{a}$

\begin{tabular}{lccccccc}
\hline$\dot{m}_{j} / \dot{m}_{0}$ & $\begin{array}{c}F_{j y}, \\
\text { and N }\end{array}$ & $\begin{array}{c}F_{w y}, \\
\text { and N }\end{array}$ & $\begin{array}{c}\sum F_{y}, \\
\text { and N }\end{array}$ & $\begin{array}{c}F_{x}, \\
\text { and N }\end{array}$ & $C_{\mathrm{AV}}$ & $C_{\mathrm{AF}}$ & $C_{\mathrm{AI}}$ \\
\hline 0.008 & 0.672 & 1.202 & 1.898 & 134.29 & 1.012 & 1.002 & 0.994 \\
0.025 & 2.936 & 3.150 & 6.086 & 134.68 & 1.015 & 1.005 & 0.981 \\
0.038 & 4.569 & 4.001 & 8.570 & 135.03 & 0.955 & 1.009 & 0.972 \\
0.051 & 6.158 & 5.031 & 11.189 & 135.61 & 0.925 & 1.014 & 0.965 \\
0.063 & 7.755 & 6.033 & 13.788 & 135.90 & 0.921 & 1.018 & 0.958 \\
0.076 & 9.341 & 7.134 & 16.475 & 136.21 & 0.904 & 1.024 & 0.952 \\
0.089 & 10.976 & 8.170 & 19.146 & 136.47 & 0.897 & 1.027 & 0.943 \\
0.102 & 12.577 & 9.173 & 21.75 & 136.75 & 0.885 & 1.032 & 0.936 \\
0.127 & 15.792 & 11.12 & 26.908 & 137.32 & 0.872 & 1.043 & 0.925 \\
\hline
\end{tabular}

${ }^{\mathrm{a}} N P R=37.5, F_{0}=134.05 N \pm 0.5 \%, I_{\mathrm{sp}}^{0}=57.9 \mathrm{~s}, \dot{m}_{0}=236.2 \mathrm{~g} / \mathrm{s}$.

growth in region 1 that ends with the plateau pressure that is governed by the primary upstream vortex in region 2 . After the plateau zone, and a smaller descent between the primary and secondary upstream vortices, the pressure rapidly increases to the pressure peak value in region 3 , which is dominated by the secondary upstream vortex. After reaching the maximum peak, it steeply decays with a staircaseshaped secondary peak caused by the interferences at the edge of the secondary injection port. Downstream of the injection port, after a slight pressure rise due to the injection port trailing edge, there is a widely distributed low-pressure zone in region 4 . A weak but large pressure hump is closing the zone of region 5, ending the sequence with the trailing edge of the primary downstream vortex and the boundary-layer reattachment.

\section{B. Gas Flow Properties}

\section{Secondary Flow Conditions}

The secondary flow chamber pressure was set to be in the range of primary flow conditions with the intention to simulate the system without additional gas supply and pressurization. With the defined secondary injection geometry, the $\mathrm{SPR}=1$ condition yielded a secondary-to-primary mass-flow-rate ratio of $8 \%$. Further altering of the SPR, and thus the modified mass flow ratio, affects the secondary injection reactive force and penetration height. Shortening of the detachment distance and decrease of the whole separation zone are detected for SPR $=0.833$ when comparing the experimental and numerical pressure data for two SPR modes in Figs. 15 and 16. Both the Kulite sensors and the Scanivalve pressure scanner identified a pressure rise in the separated flow zone and its downstream evolution. In shown figures, Kulite micro-transducers pressure results are presented with error bars, while the Scanivalve pressure results are represented with triangle symbols. The pressure drop behind the injection (depicted at profiles from meridians 1,2, and 3) appears to be unaffected by the change of SPR, which is not the case for the wallpressure profiles after meridian 4. By lowering the SPR value, we can observe a decrease in the plateau pressure levels. As the whole separation zone is strongly affected by the adverse pressure gradient, a smaller SPR, and therefore a drop in the APG and horseshoe vortex evolution, will shorten the upstream separation distance. As assumed and evaluated from the adopted analytical model, the experimental and numerical results verify that the upstream separation and plateau levels in the symmetry plane accordingly change with every SPR step, which can be observed in Fig. 17. Variation of the injection rate directly governs the secondary injection thrust force rate. However, the relation between the injection rate and the interaction wall side force component is not completely linear. Namely, as seen in the tabulated force data in Tables 3 and 4, the contribution level of the secondary injection reactive force-momentum $F_{j y}$ component and the interaction force $F_{w y}$ component exerted on the main nozzle wall varies with the change of the secondary injection rate. For a very small injection rate, the contribution of the interaction force component is higher than the one coming from the secondary injection momentum itself. The low momentum and secondary mass flow rate, which figure in the divider of performance coefficients, result in higher performance rates reaching the unit value. In Fig. 18, we can observe the evolution of performance amplification factors that vary with the injection regime from the very low injection case to the high secondary to primary injection rates. At a low injection rate, at which the main flow pressure influence on the injection port is strong, $C_{\mathrm{AV}}$ is steady around the unit until the injection rate ratio reaches $3 \%$ of the main mass flow. With the increase of injection rate, $C_{\mathrm{AV}}$ drops until a mass-flow-rate ratio of $5 \%$ and the amplification plateau value is observed between 5 and $6.5 \%$ of the injection rate ratio. With further increase in injection, $C_{\mathrm{AV}}$ decays. This complies well with [23] and in [44] on 2-D nozzle slot injection, where a higher injection-performance ratio is noticed for the injection rate ratios between two and four.

Observation of the evaluated results suggests that a small to moderate injection rate is fuel-consumption favorable, whereas for the higher injection rates, a certain sacrifice of the regular performance is needed in order to obtain higher vectoring angles.

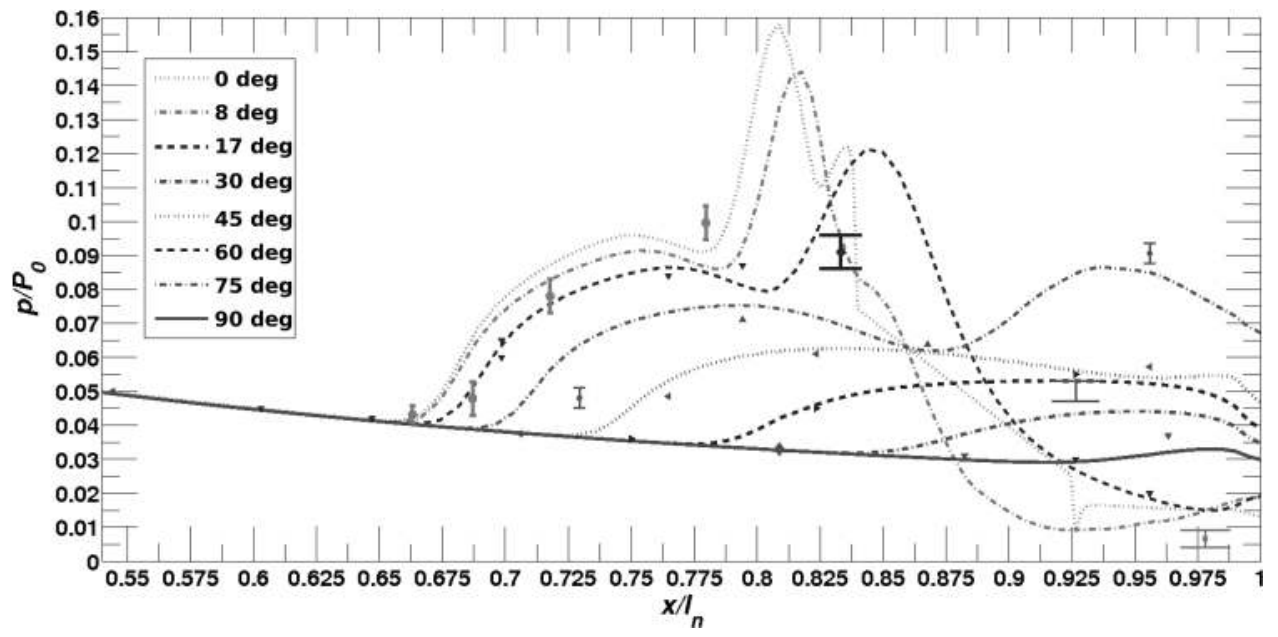

Fig. 15 Wall-pressure at meridional positions around the nozzle axis for SPR $=1$ (lines - CFD, symbols - experiment). 


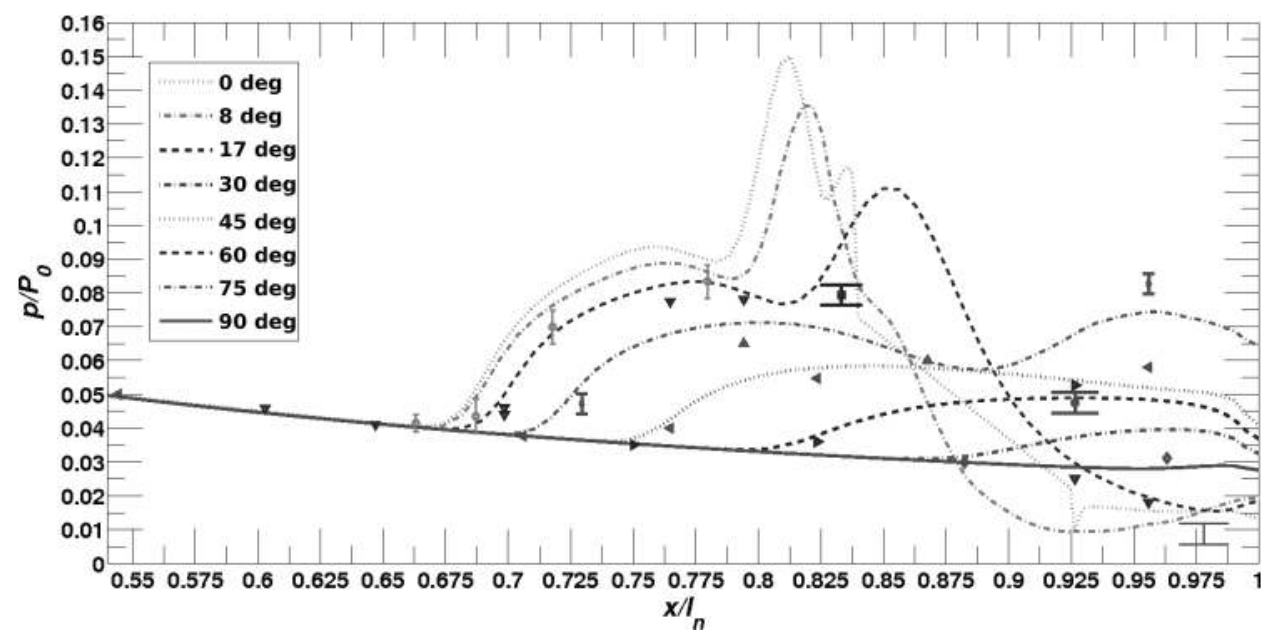

Fig. 16 Wall-pressure at meridional positions around the nozzle axis for SPR $=0.833$ (lines - CFD, symbols - experiment).

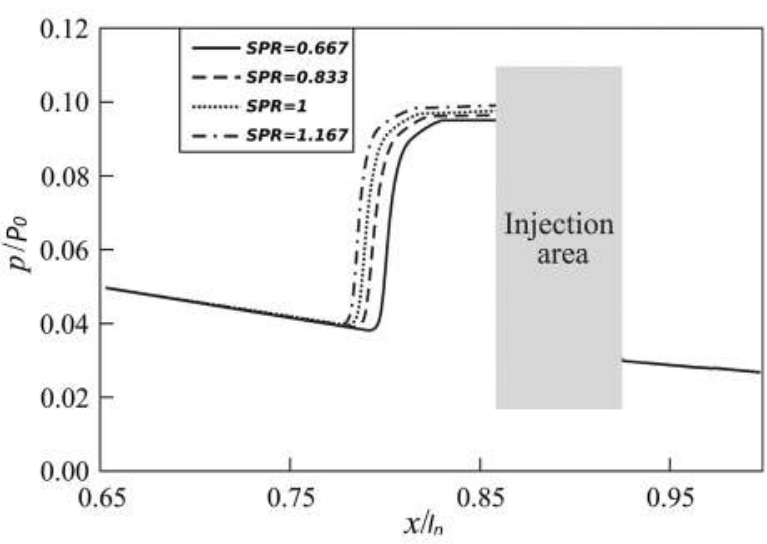

a) Analytically evaluated profiles

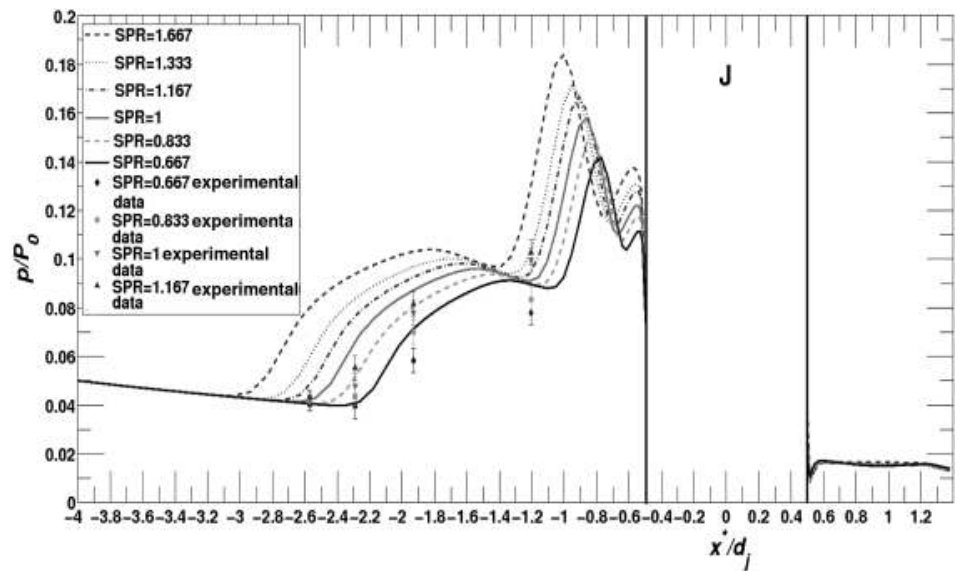

b) Experimental-numerical results

Fig. 17 Wall-pressure at injection side of symmetry plane for different SPRs (lines - CFD, symbols — experiment).

\section{Primary Flow Conditions}

Moreover, the primary nozzle pressure ratio mainly affects the downstream portion of the crossflow interaction field. Increase of ambient conditions over the adaptation values leads to the shortening of a normal flow recompression sequence. Sequentially, the further increase of ambient pressure results in the occurrence of a Mach disk at the nozzle exit section. This $\lambda$ shock-wall interaction is further altered by the nature of the established shock and crossflow field interface.

In Fig. 19, several characteristic overexpansion modes are identified, consulting the numerically and experimentally obtained schlieren snapshots. As experimental schlieren captures only the jet

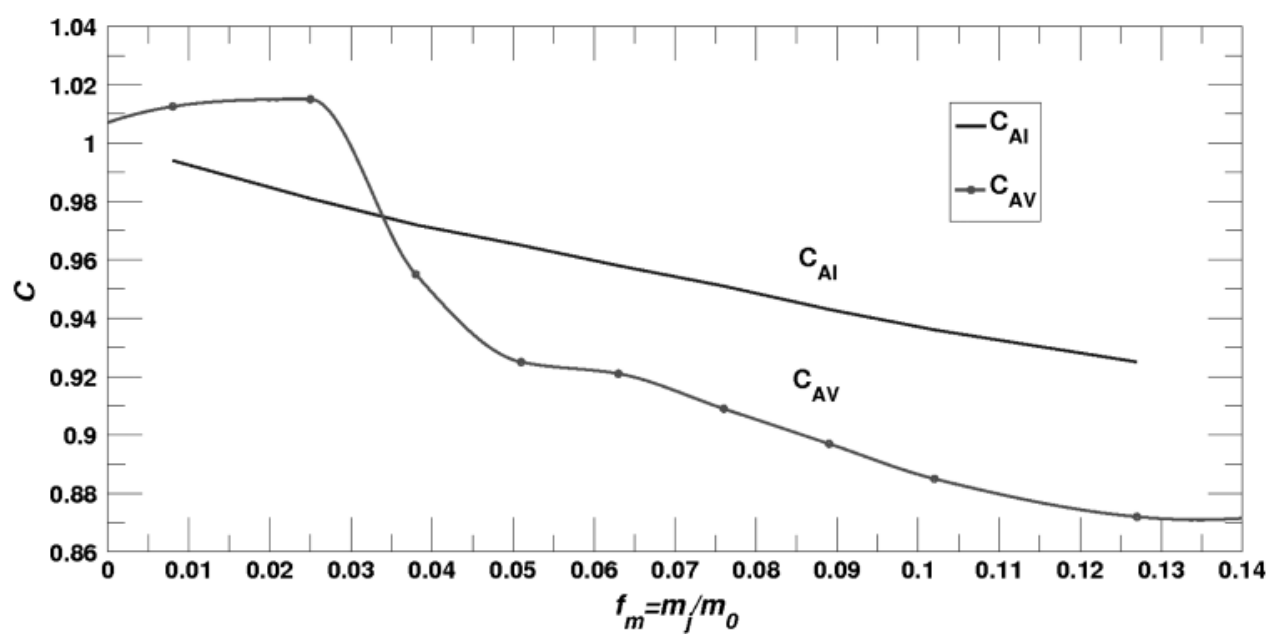

Fig. 18 Vectoring and specific impulse amplification factor vs mass-flow-rate ratio. 
exterior part at the nozzle exit; the interaction zone is shown in more detail, depicted on the numerical plots. Reduction of the nozzle pressure ratio (NPR) to $50 \%$ of $N P R_{D}$ leads to the initial separation at the nozzle exit. an increase of ambient pressure above the adaptation level initially may favorably affect the fluidic vectoring system performances. Namely, inflow from the exterior and an increase of pressure at the nozzle lip confront the low-pressure region behind the injection and its suction effect. Acting as a relief mechanism for the injection plume, this is found to allow higher penetration while the thrust force decreases with overexpansion and leads to the higher pitch vector angles. However, at $40 \%$ of $N P R_{D}$, an overexpansion leads to the prominence of a Mach disk reflection phenomenon close to the nozzle lip. Further upstream, movement of the Mach disk inside the nozzle largely influences the SITVC system through the separation at the opposite nozzle side, whereas the injection side is filled with the injection plume. The SITVC becomes completely inoperable with the Mach disk appearance at the injection cross section. However, in some configurations, inflow from all injection positions may help in dealing with the effect of separated nozzle flow [45]. Coupled experimental and numerical data for different

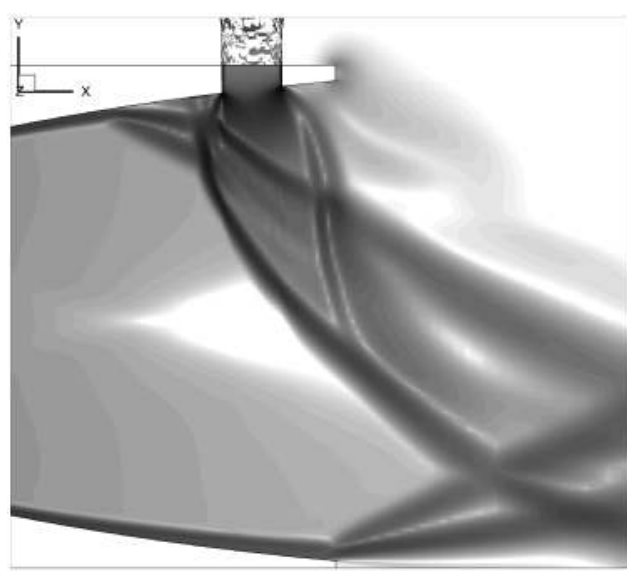

a) $\mathrm{NPR}=20, \delta=7.57 \mathrm{deg}$ numerical schlieren

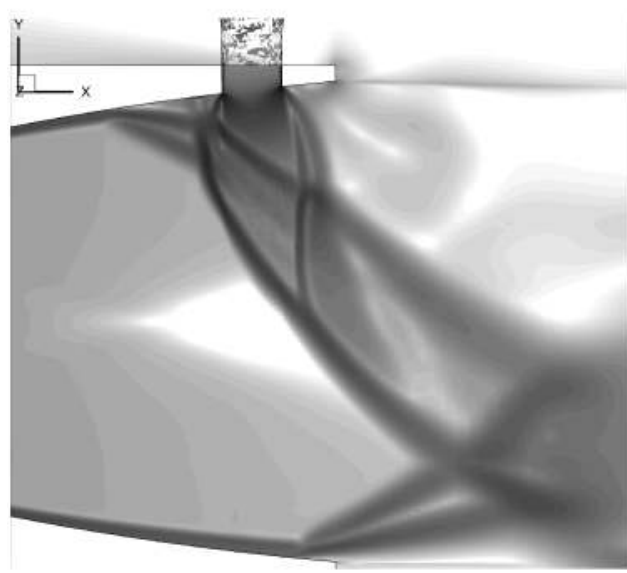

c) NPR $=15, \delta=8.08$ deg numerical schlieren

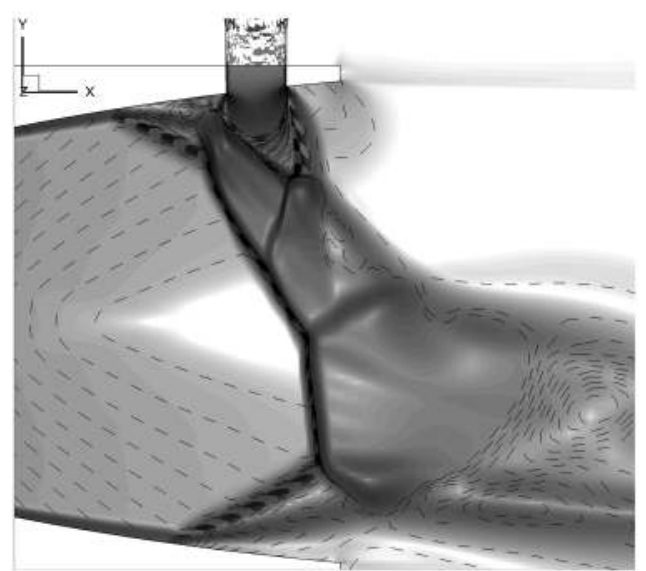

e) $\mathrm{NPR}=\mathbf{1 0}, \delta=\mathbf{5 . 6 8}$ deg numerical schlieren with contours

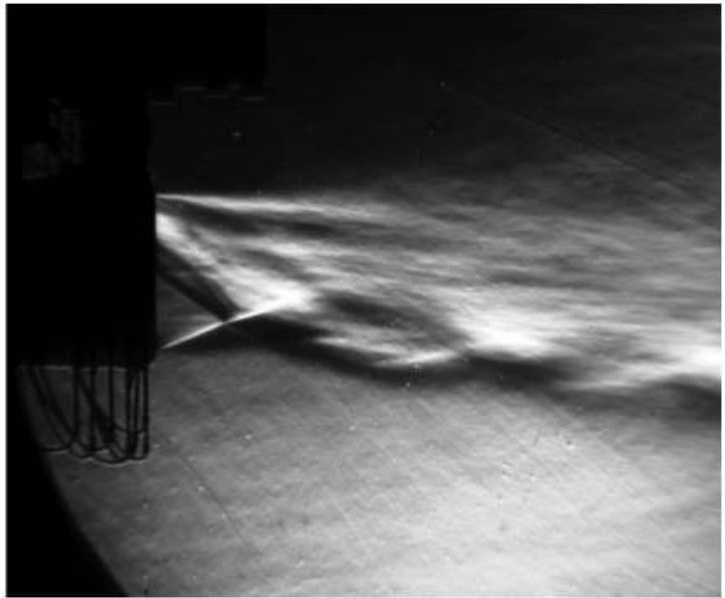

b) $\mathrm{NPR}=\mathbf{2 0}$ experimental $\mathrm{Z}$ schlieren

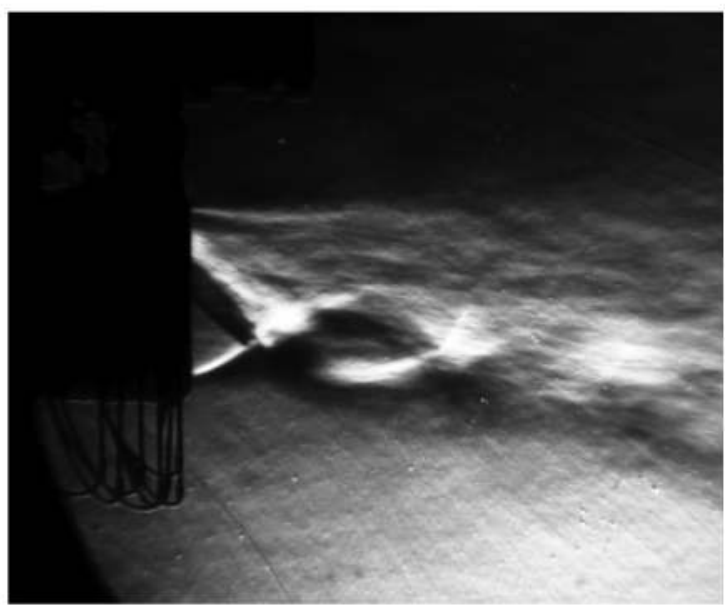

d) $\mathrm{NPR}=15$ experimental Z schlieren

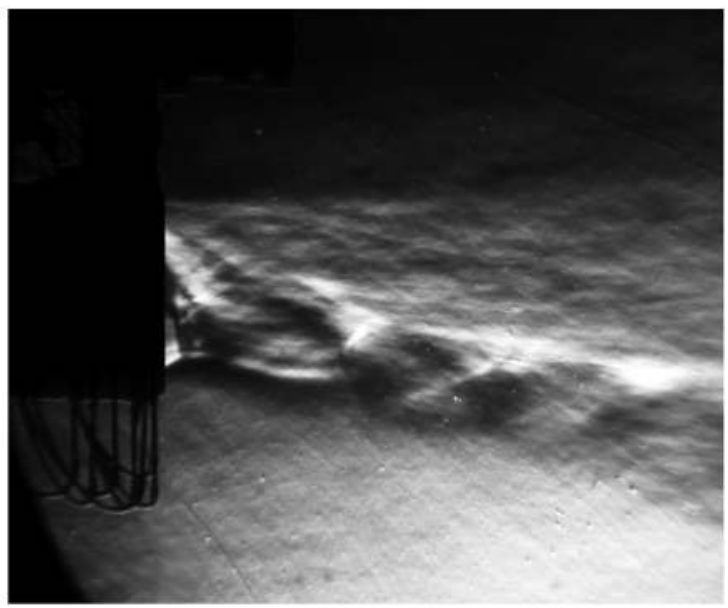

f) $\mathrm{NPR}=10.4$ experimental $\mathrm{Z}$ schlieren

Fig. 19 Numerical and experimental schlieren photographs for SPR $=1$ and series of overexpansion NPRs. 


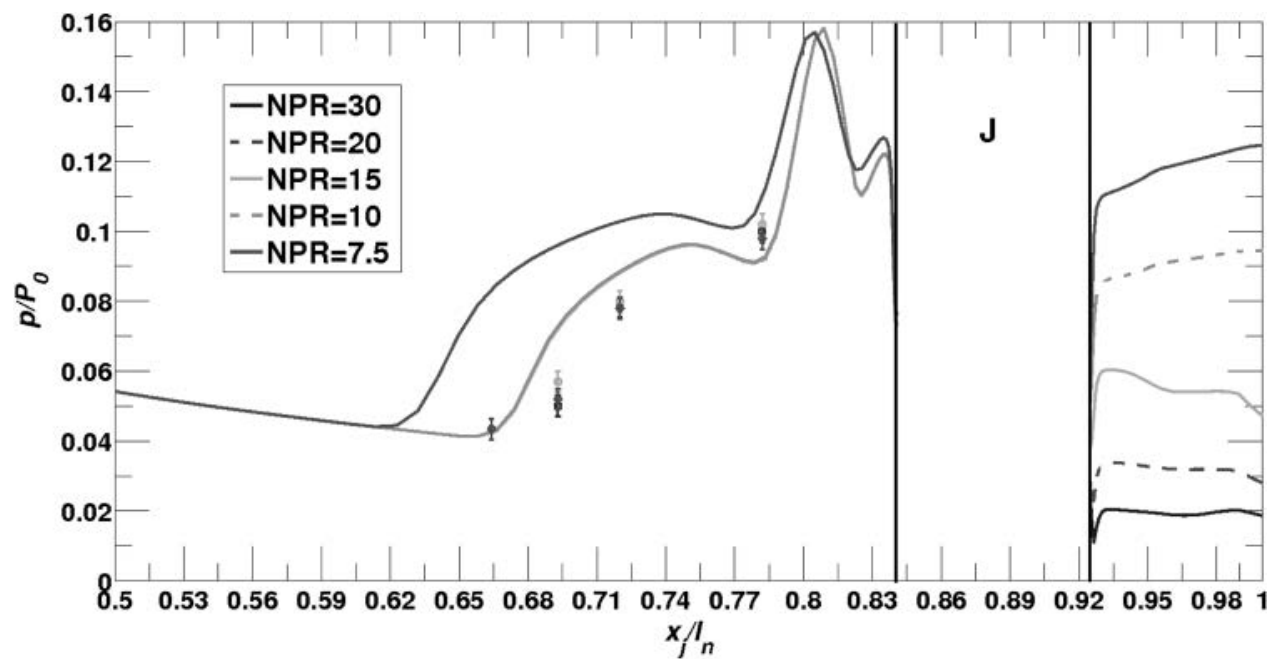

Fig. 20 Wall-pressure profiles for constant SPR $=1$ and series of overexpansion NPRs of TIC-i nozzle (lines - CFD, symbols - experiment).

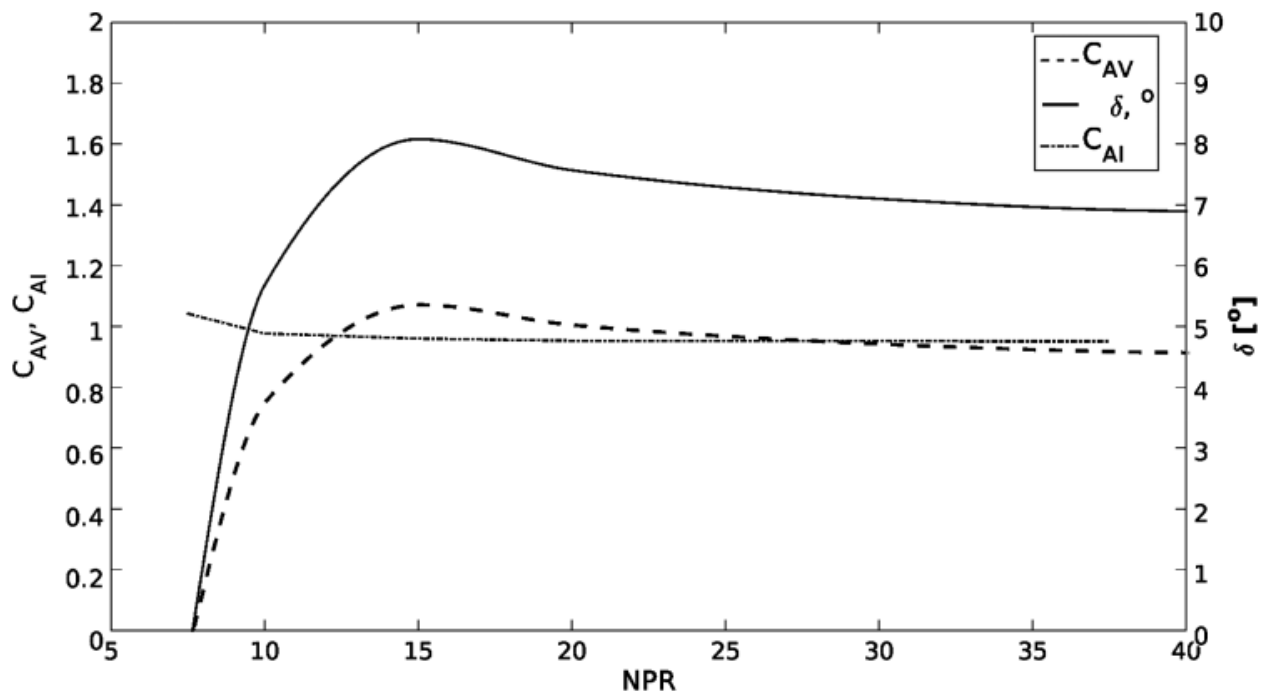

Fig. 21 Performance parameters and deflection angle plotted versus NPR for TIC-i: $x_{j} / l_{n}=0.882$ nozzle and SPR $=1$.

overexpansion cases can be observed in Fig. 20. Only wall-pressure profiles aft of the injection port are affected by the main NPR change. Some moderate increases of pressure in the low-pressure suction zone aft of the injection port relieve the action on the secondary jet, which allows a slight increase in vectoring amplification. In an analysis of tested cases, an optimal nondimensional $C_{\mathrm{AV}}$ value is reached around $N P R \sim 15$, which corresponds to a maximal vector pitch angle $\delta$, as shown in Fig. 21. A further NPR decrease largely deteriorates the vectoring performance and leads to performance decay.

\section{Intrinsic Gas Properties}

In addition to a flow conditions analysis, the intrinsic gas properties of involved species are found to be crucial for the characterization of a fluidic vector system performance. Experiments with the several secondary injectant gas species were accompanied by the numerical simulations, which together emphasized the important effect of thermodynamic properties on this wall-bounded crossflow system. Evaluated result data of the current investigation highlighted the important influence of secondary to primary ratios of molecular weights and specific heats on the flow deflection. In addition, molecular viscosity and diffusivity were found to have only a minor effect on the separation of the viscous boundary layer and shock interaction interface.
The results tabulated in Tables 5 and 6 show that the efficiency of a fluidic thrust vector control system largely increases with the low secondary to primary $\gamma M_{g}$ ratios. This can be justified by considering the higher sonic velocity characteristic of a penetrating gas into the main flow, whereas no important mixing is assumed in the first part of interaction [13,32]. Observing the performance graphs in Figs. 22 and 23 , it can be observed that this dependency is especially significant regarding the consumption efficiency of the rocket engine SITVC operation. In the reported cold-flow experiments, a secondary

Table 5 Multispecies injection experimental statistical average data

\begin{tabular}{lcccccc}
\hline $\begin{array}{l}\text { Second } \\
\text { species }\end{array}$ & $f_{m}$ & $\gamma_{j}$ & $M_{g}$ & $F_{y}, \mathrm{~N}$ & $F_{x}, \mathrm{~N}$ & $\delta, \mathrm{deg}$ \\
\hline \multicolumn{7}{c}{ Constant $\mathrm{SPR}=1$} \\
Air & 0.076 & 1.4 & 28.96 & $16.17 \pm 2 \%$ & $136.1 \pm 0.5 \%$ & $6.78 \pm 4 \%$ \\
Helium & 0.030 & 1.667 & 4.0026 & $16.95 \pm 2 \%$ & $136.1 \pm 0.5 \%$ & $7.1 \pm 4 \%$ \\
$\begin{array}{l}\text { Carbon } \\
\text { dioxide }\end{array}$ & 0.092 & 1.297 & 44.01 & $16.20 \pm 2 \%$ & $136.2 \pm 0.5 \%$ & $6.78 \pm 4 \%$ \\
Argon & 0.096 & 1.663 & 39.948 & $16.05 \pm 2 \%$ & $136.3 \pm 0.5 \%$ & $6.72 \pm 4 \%$ \\
\multicolumn{7}{c}{ Constant $f_{m}=0.076$} \\
$\begin{array}{l}\text { Carbon } \\
\text { dioxide }\end{array}$ & 0.833 & 1.297 & 44.01 & $13.76 \pm 2 \%$ & $136.1 \pm 0.5 \%$ & $5.77 \pm 4 \%$ \\
Argon & 0.803 & 1.663 & 39.948 & $12.95 \pm 2 \%$ & $136.3 \pm 0.5 \%$ & $5.43 \pm 4 \%$ \\
\hline
\end{tabular}


Table 6 Multispecies injection numerical averaged force and performance data: constant $f_{m}=\mathbf{0 . 0 7 6}$

\begin{tabular}{llrrrccc}
\hline Case & $\mathrm{SPR}$ & $F_{i v}, \mathrm{~N}$ & $F_{w v}, \mathrm{~N}$ & $\delta, \mathrm{deg}$ & $C_{\mathrm{AV}}$ & $C_{\mathrm{AF}}$ & $C_{\mathrm{AI}}$ \\
\hline Air & 1 & 9.473 & 7.134 & 6.89 & 0.904 & 1.024 & 0.952 \\
Helium & 2.534 & 24.279 & 14.972 & 16.20 & 2.124 & 1.049 & 0.975 \\
Carbon dioxide & 0.833 & 7.713 & 6.254 & 5.87 & 0.904 & 1.019 & 0.947 \\
Argon & 0.803 & 7.636 & 5.426 & 5.49 & 0.719 & 1.017 & 0.945 \\
\hline
\end{tabular}

injection of helium into dry air was largely superior to those injections of more inert gases as argon or carbon dioxide. The observed secondary to primary gas species intrinsic properties relation comply well with the remarks of an efficiency increase in use of highenthalpy reactive injectant gases in [32] and others.

\section{Geometric Aspects of SITVC}

Geometric parameters that mainly influence SITVC system performances were investigated in regard to the optimization of primary and secondary nozzle aspects. The position of the secondary injection along the divergent section of the main nozzle and its inclination toward the main axis were found to represent the substantial factors in a SITVC system design. In addition, limited amelioration of SITVC was possible by applying optimization to the contour design of primary and secondary nozzle.

\section{Secondary Injection Axial Position}

The position of secondary injection at the divergent section of a rocket propulsive nozzle was investigated on a conical nozzle model with constant contour slope. Two experimental models with injections placed at 90 and $70 \%$ of the main nozzle length were considered, as reported in [25]. The experimental analysis was complemented by the numerical simulations of different injection positions using the same conical nozzle model. Two experimental cases differed by the appearance of the shock reflection inside the nozzle and the upstream and downstream sizes of the separated region. In the first case of injection at $x_{j} / l_{n}=0.7$, in Fig. 24a, the bow shock propagated through the nozzle and reflected from the opposite wall side, affecting the complete flow in the cross section. In the vicinity of the reflection zone, opposite the injection port, the boundary layer separated, forming a recirculation bubble that, in the given case, closed at the nozzle lip. The shock reflection inside the SITVC nozzle, as shown in Fig. 25, had a strong effect on the flow deflection and vectoring rate. Displacement of the injection point closer to the nozzle lip, as in the case of injection at $x_{j} / l_{n}=0.9$ in Fig. 24b, prevented the occurrence of the shock reflection inside the nozzle, allowing the injectant plume and horseshoe vortex to exit the nozzle domain unaffected, whereas the interaction took place in the exterior domain.

Transverse injection at the positions closer to the nozzle lip also reduces the low-pressure zone aft of the injection port, allowing deeper penetration and separation farther upstream. This also can be observed in the skin-friction profiles presented in Fig. 26, showing the separation distance augmentation with each consecutive displacement of the injection point toward the nozzle exit.

Analysis of the acquired force data, which are also given in Table 7, and performance graphs in Fig. 27 indicates that the injection positions closer to the nozzle exit provide a cleaner crossflow interaction

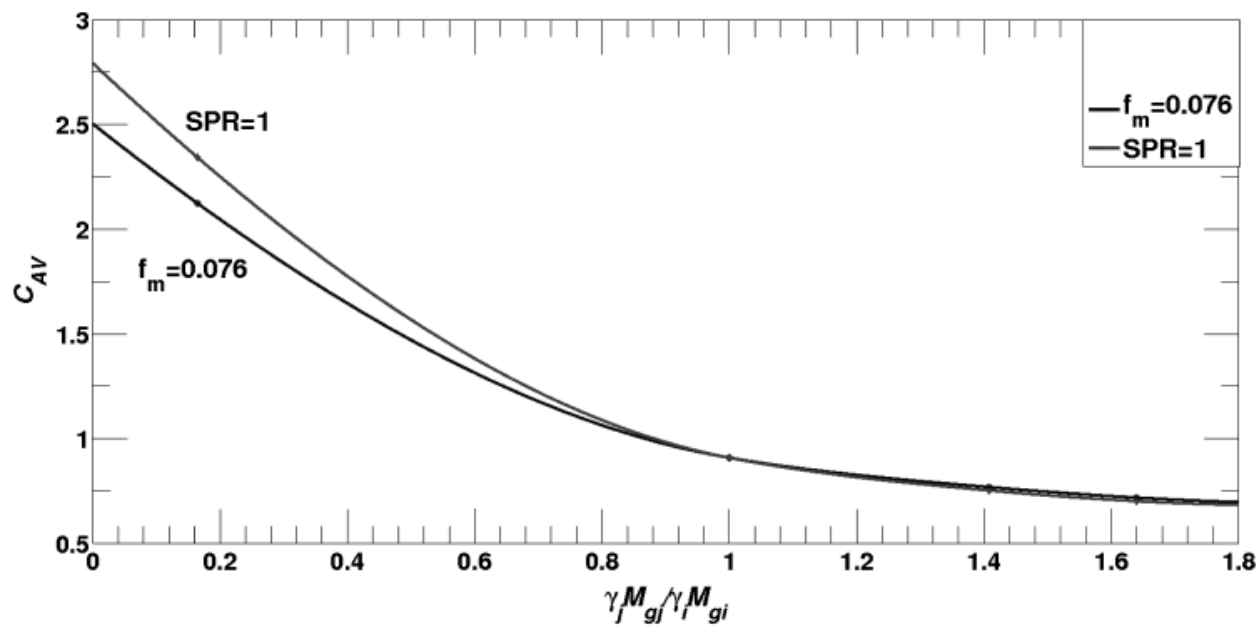

Fig. 22 Vectoring amplification versus $\gamma M_{g}$ product ratio of injectant in the main flow.

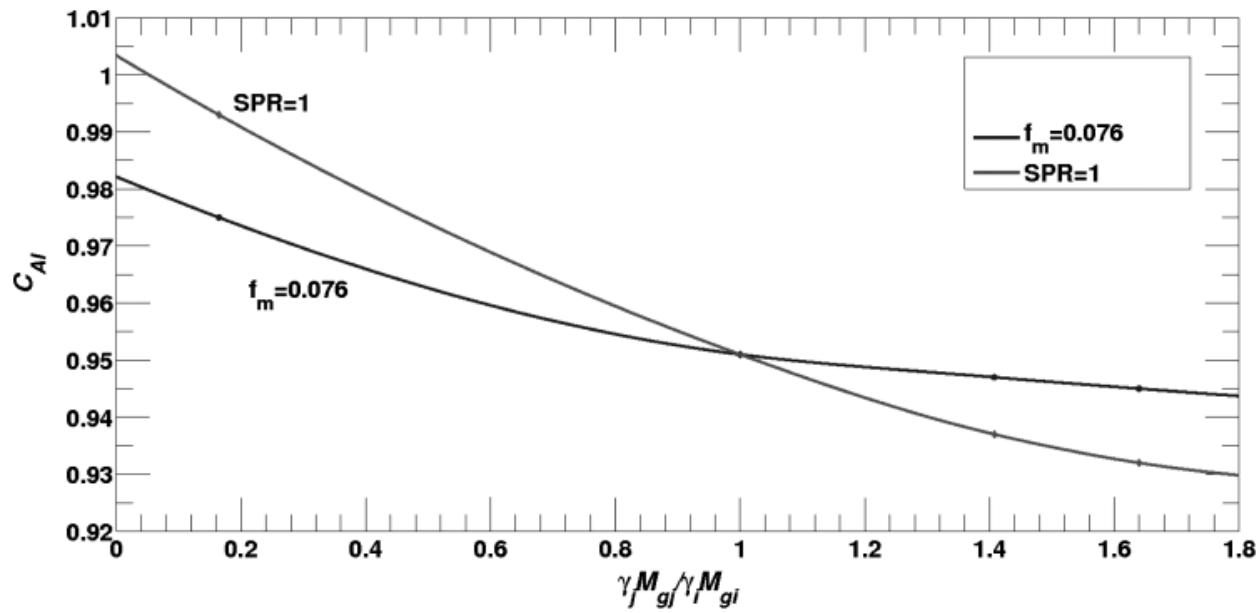

Fig. 23 Specific impulse amplification versus $\gamma M_{g}$ product ratio of injectant in the main flow. 


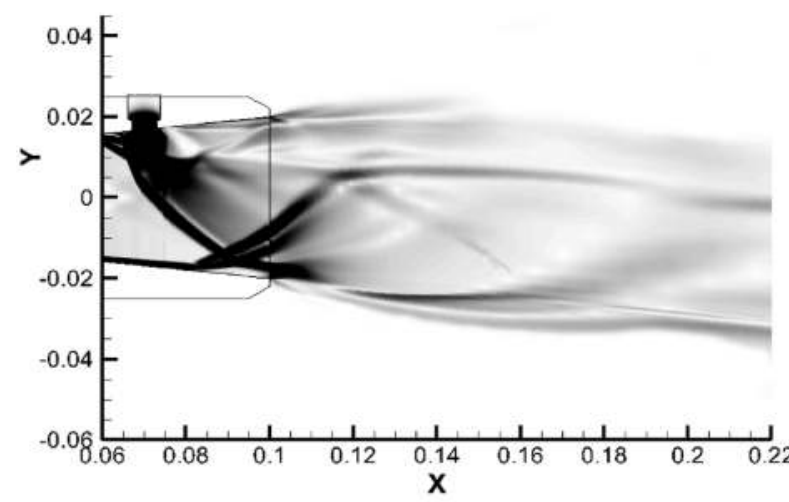

a) $x_{j} / l_{n}=0.7$

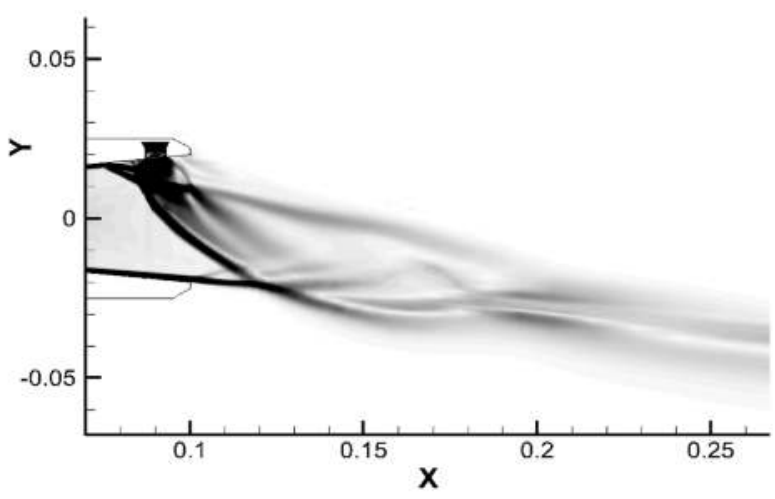

b) $x_{j} / l_{n}=0.9$

Fig. 24 Numerical schlieren images of SITVC conical nozzle with two injection positions.

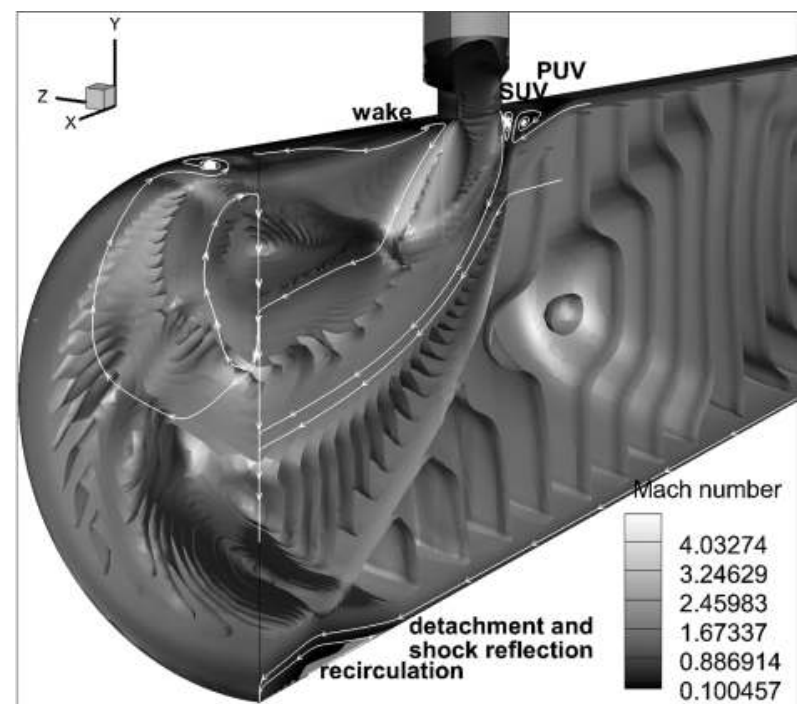

Fig. $25 x_{j} / l_{n}=0.7$ case 3-D flowfield with iso-Mach contours.

inside the nozzle with an increased jet deflection rate. Two distinctive zones of secondary injection placements can be identified as SITVC with and SITVC without bow-shock reflection inside the nozzle domain. As can be seen in the given data, cases where shock-wall reflection occurs strongly deteriorate vector deflection. For the given test nozzle model, a limiting case is found for the injection point at $x_{j} / l_{n}=0.75$, where the bow shock impinges on the outer nozzle lip. From this injection position towards the nozzle exit, deflection linearly increases due to a gradual rise of the high-pressure zone upstream of the injection port and a decline of the downstream low-pressure zone. In regard to our intended use of a second-stage launcher, the optimal position of $x_{j} / l_{n}=0.9$ is selected and used in further analyses.

\section{Secondary Injection Angular Position}

Further optimization of the SITVC nozzle geometric characteristics considers the injection attitude vector. As reported in [46], the SITVC performance rate gradually deteriorates with the secondary injection inclined downstream in reference to the main axis, whereas the upstream inclination considerably augments the produced side force. Some researchers, as in [45], even proposed usage of a gimbaled secondary injection for a larger operation envelope.

In the current investigation, a 20 deg upstream inclined injection was experimentally tested using the conical nozzle model and compared to the results of numerical simulations. Subsequently, angular inclination of a secondary injection was investigated numerically on the range of upstream inclined angles with a $10 \mathrm{deg}$ step.

As observed in Fig. 28, the main action of the upstream inclined injection is an additional blockage to the oncoming supersonic flow. This additional push by the windward side of the secondary plume yields a steeper slope gradient in the bow shock, and thus in the flow deflection evolution, and it effectively aspirates the trapped flow in the separation zone through the enlarged interface. Consequently, the interaction wall side force component increases, which leads to a higher side force magnitude.

Contrary to the favorable deflection rate, the steeper bow shock and oppositely directed axial component of the secondary injection reaction force a decrease of the main nozzle efficiency in terms of thrust force.

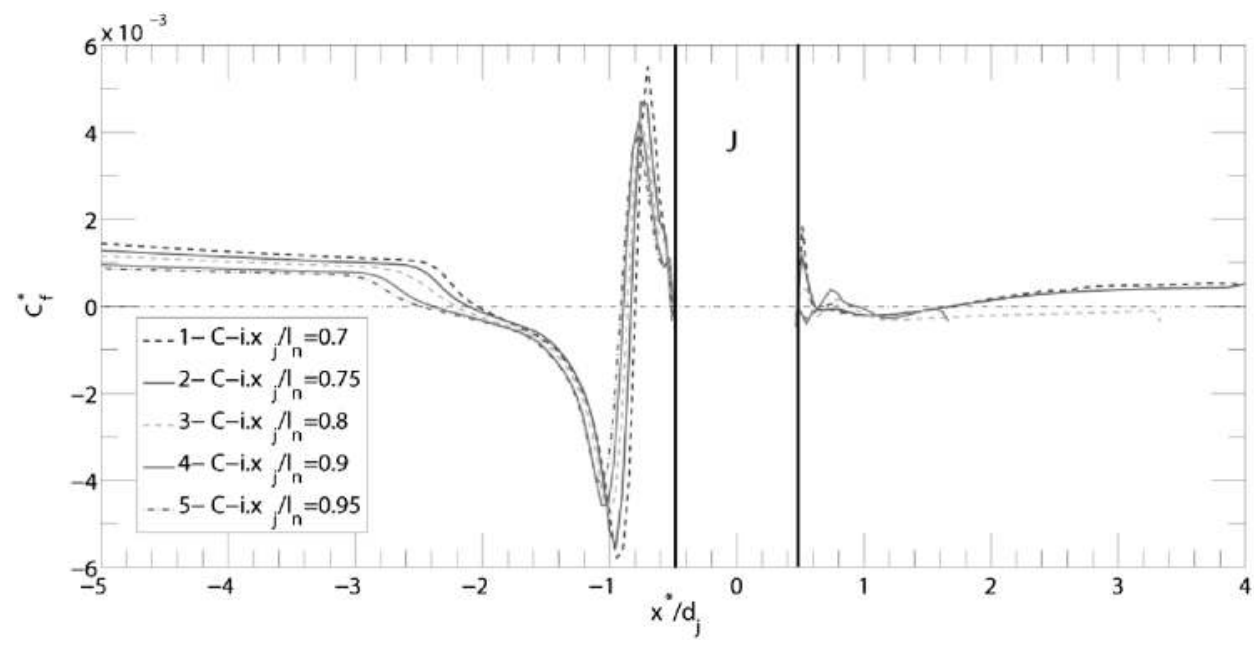

Fig. 26 Skin-friction profiles in symmetry plane for different injection positions. 
Table 7 Force and performance metrics data at different injection positions of conical nozzle test case ${ }^{a}$

\begin{tabular}{lclllll}
\hline Inclination case & $F_{j v}, \mathrm{~N}$ & $F_{w v}, \mathrm{~N}$ & $\delta, \mathrm{deg}$ & $C_{\mathrm{AV}}$ & $C_{\mathrm{AF}}$ & $C_{\mathrm{AI}}$ \\
\hline$x_{j} / l_{n}=0.7$ & -8.04 & 9.91 & 0.82 & 0.1 & 1.037 & 0.958 \\
$x_{j} / l_{n}=0.75$ & 0.013 & 9.914 & 4.6 & 0.554 & 0.97 & 0.896 \\
$x_{j} / l_{n}=0.8$ & 6.308 & 9.92 & 7.15 & 0.861 & 1.03 & 0.951 \\
$x_{j} / l_{n}=0.9$ & 8.67 & 9.94 & 8.27 & 0.976 & 1.017 & 0.939 \\
$x_{j} / l_{n}=0.95$ & 8 & 9.94 & 8.01 & 0.963 & 1.018 & 0.94 \\
\hline${ }^{\mathrm{a}} N P R=37, S P R=1, f_{m}=0.083, F_{0}=126.41 \mathrm{~N}, I_{\mathrm{sp}}^{0}=57.77 \mathrm{~s}$.
\end{tabular}

As illustrated in Fig. 29, upstream separation increases with the each consecutive step, which is also evident in the extracted wallpressure profiles. The increase of the upstream inclination value also modifies the injection port area, which is found in the intersection of the sonic throat and the divergent nozzle wall. The injection port area enlargement leads to a nonuniform pressure distribution, as observed in Fig. 30 at the injection port, which is prone to backpressure effects.

Even though a breakdown angular value was not reached in terms of produced side force, we can observe, for the $\phi=60$ deg case, a depicted pressure plot in Fig. 30b that shows high pressure in the upstream zone strongly affects a preexpanded secondary flow at the windward portion of the secondary injection port section. This leads to a potentially unsteady motion of the separated flow as observed in probing of the velocity and force.

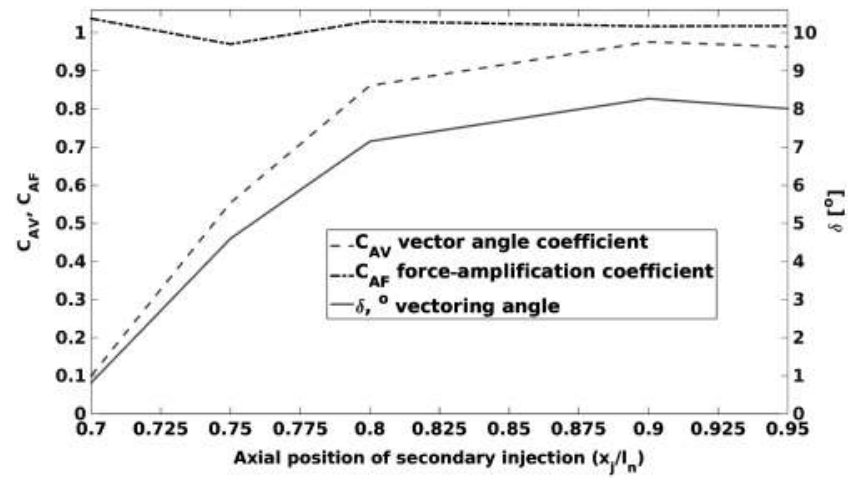

Fig. 27 Evolution of vectoring and force-amplification factors at different injection positions.

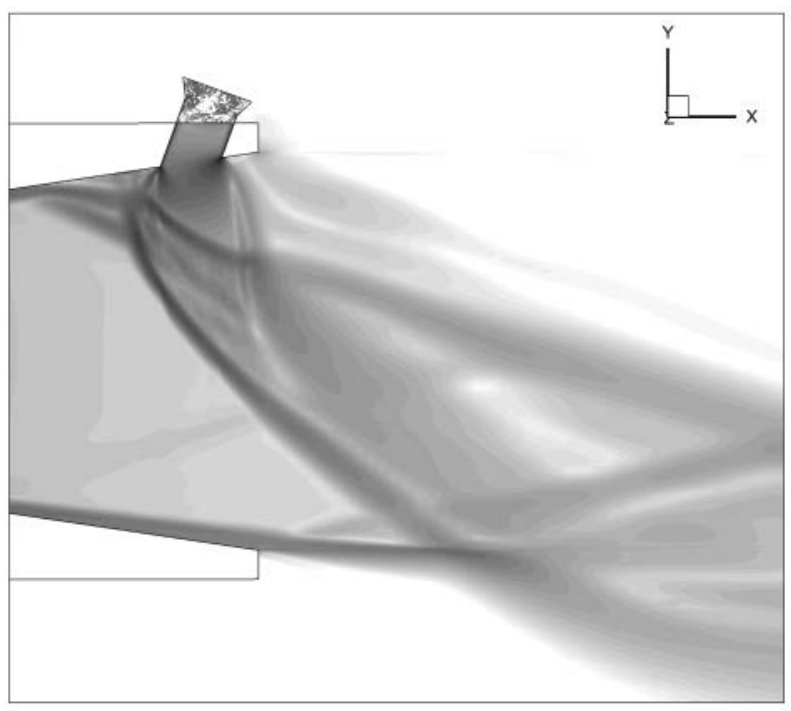

a) Numerical schlieren in symmetry plane
The force data evaluated from the investigated cases (Table 8) indicate that the interaction side force component is directly related to the amount of inclination. Accordingly, with an upstream angular attitude increase, the contribution of the side force component from the crossflow interaction surpasses the contribution of the secondary injection reactive force normal component. Additionally, evaluated test cases indicate that angles larger than $\phi>40 \mathrm{deg}$ gradually produce a very complex and deviated flowfield with detrimental effects on the axial force, side loads, and nozzle stability. Optimizing between these parameters, an optimal upstream inclination is found in selected test cases around $27 \mathrm{deg}$.

\section{Secondary Injection Nozzle Geometry}

The geometry design of a secondary injection may additionally affect the crossflow field generation and the separation mode in main flow. With the imposed conditions of the same secondary mass flow rate and chamber pressure, a rectangular slot-type injection nozzle was designed. The longer edge of the rectangle is defined as a circular arc over the central angle, where the center is defined at the selected position of the main nozzle axis. The shorter edge is then found from the condition of an identical throat area, as illustrated in Fig. 31. Following the research reported in [24,44], which dealt with the multiple-slot central angle sizes, the central angle value of $\theta=$ $30 \mathrm{deg}$ is selected as an optimal value considering the pressure rise in the separation zone:

$$
a=2 \pi R_{C j}\left(\frac{\theta}{360}\right), \quad a_{j}=2 R_{C j} \sin \left(\frac{\theta}{2}\right), \quad b_{j}=A_{j} / a_{j}
$$

To avoid the stress concentration and vortex generator at the sharp corners, a slot port is shaped as a rounded rectangle over the central arc of $\theta=30 \mathrm{deg}$. The fully equipped experimental model was tested under the same conditions and compared to the circular secondary injection. In Fig. 32, a comparative view of numerical and experimental extracted schlieren photographs is given from side and top views. Comparing the schlieren snapshots of circular injection case in Fig. 11 and of slot type in Fig. 32, we can observe the increase of the upstream separated flow region in the lateral direction. A wider injection zone also affects the adverse pressure gradient and earlier upstream separation. This is also evident in wall-pressure profiles in Fig. 33, where growth in the plateau pressure level, and consequently in the farther upstream detachment point, can be noticed. The rise of the plateau pressure zone is directly related to the growth of the PUV

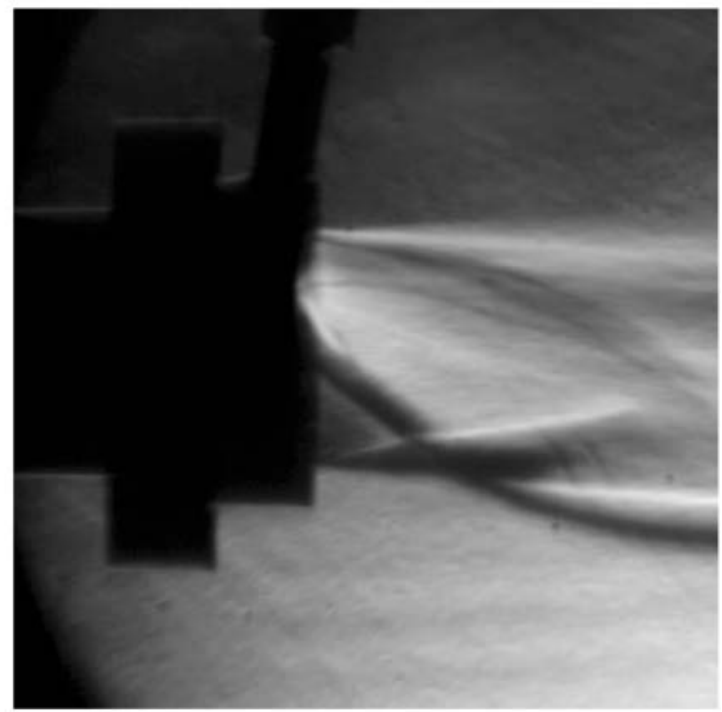

b) Z-schlieren photograph

Fig. 28 Schlieren photographs of SITVC conical nozzle with 20 deg inclined circular injection at SPR $=1$ and NPR $=37.5$ conditions, yielding $\delta=9$ deg. 


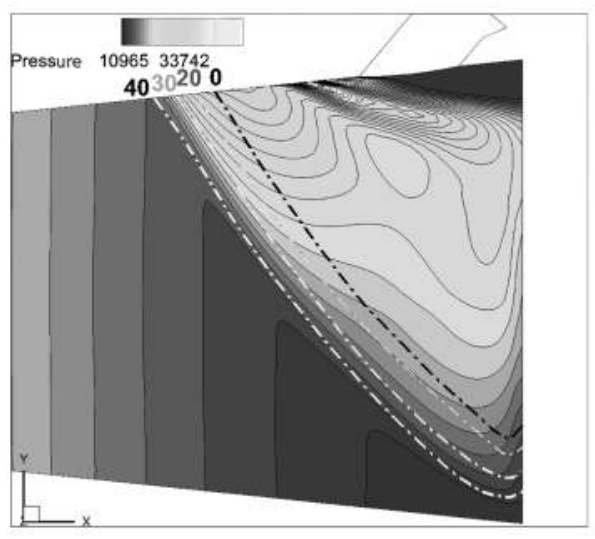

a) Pressure plot with delinations

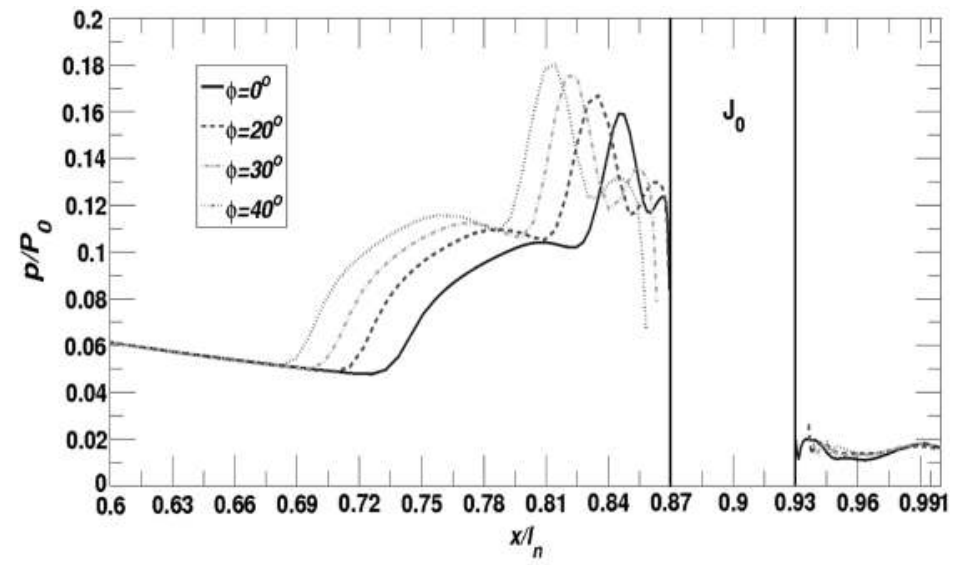

b) Profiles of different inclinations

Fig. 29 Wall pressures of inclined circular injections at $\mathrm{SPR}=1$ and NPR $=\mathbf{3 7 . 5}$ conditions.

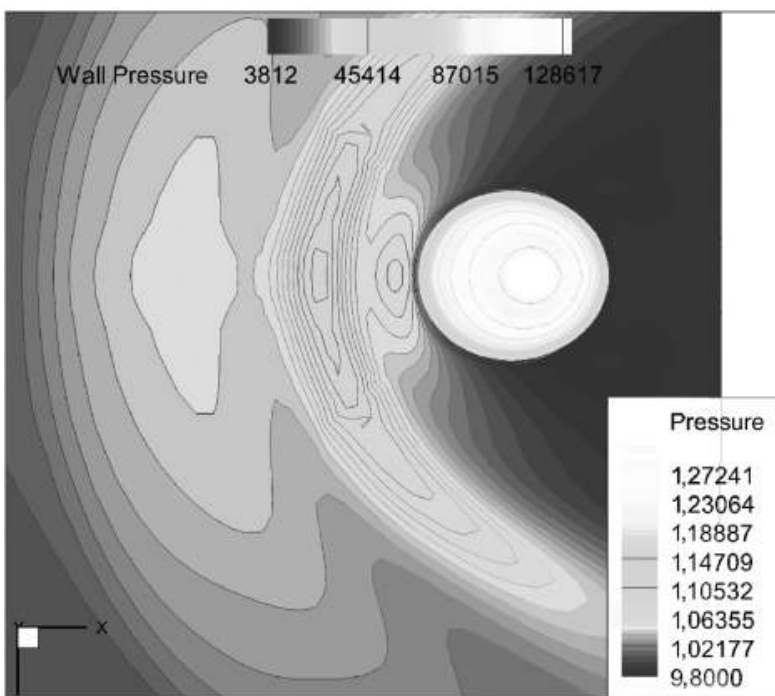

a) $\phi=20^{\circ}$

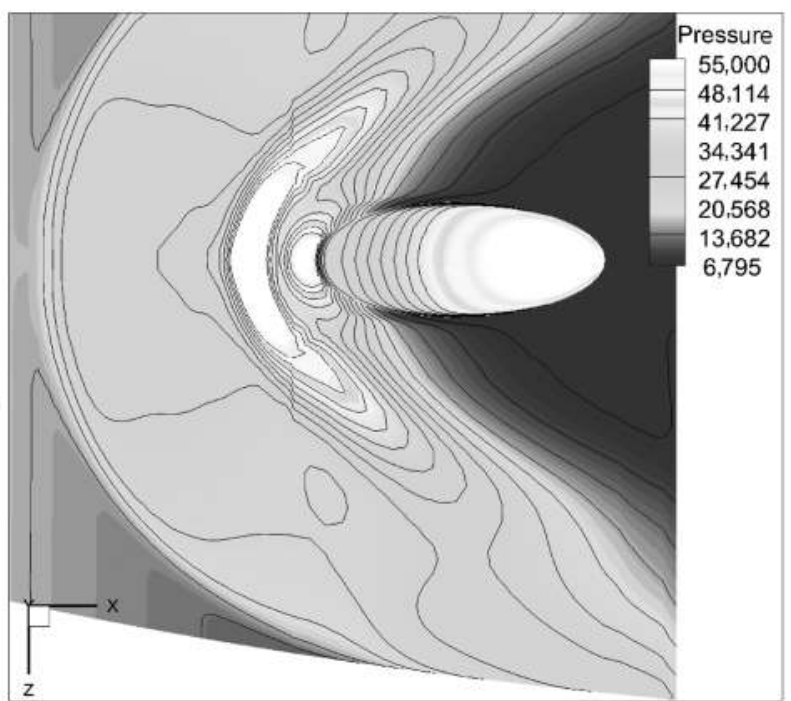

b) $\phi=60^{\circ}$

Fig. 30 Top-view wall-pressure contours at $\mathrm{SPR}=1$ and $\mathrm{NPR}=\mathbf{3 7 . 5}$ conditions.

and the corresponding horseshoe vortex. In Fig. 34, meridional pressure profiles depict the lateral evolution of increased wallpressure levels in the case of slot injection. In the given case, even the meridians of above $90 \mathrm{deg}$ are affected by secondary injection

Table 8 Force and performance metrics data of angular injection at $x_{j} / l_{n}=0.9$ conical nozzle

\begin{tabular}{lrrcccc}
\hline Inclination case & $F_{i v}, \mathrm{~N}$ & $F_{w v}, \mathrm{~N}$ & $\delta, \mathrm{deg}$ & $C_{\mathrm{AV}}$ & $C_{\mathrm{AF}}$ & $C_{\mathrm{AI}}$ \\
$\phi=0 \mathrm{deg}$ & 9.94 & 8.67 & 8.27 & 0.976 & 1.017 & 0.939 \\
$\phi=20 \mathrm{deg}$ & 10.03 & 10.28 & 9.13 & 1.088 & 1.011 & 0.933 \\
$\phi=30 \mathrm{deg}$ & 10.02 & 10.98 & 9.59 & 1.143 & 0.997 & 0.92 \\
$\phi=40 \mathrm{deg}$ & 10.05 & 11.42 & 9.97 & 1.188 & 0.98 & 0.904
\end{tabular}

${ }^{\mathrm{a}} N P R=37, S P R=1, f_{m}=0.083, F_{0}=126.41 \mathrm{~N}, I_{\mathrm{sp}}^{0}=57.77 \mathrm{~s}$. crossflow. Accordingly, the PUV- and SUV-dominated plateaus and peak pressure regions are observed in the first three reference meridians. Formation of the horseshoe vortex zone is normally detected in the vicinity of the slot's shorter side. The very good agreement of numerical and experimental pressure data is achieved in terms of the evaluation of the detachment point, the pressure rise, and the pressure peak. There is some overestimation of the plateau pressure value in $k$ - $\varepsilon$-obtained profiles, which is expected and previously observed as in [40]; but, in general, the used Reynoldsaveraged Navier-Stokes model performance was highly satisfactory. In the given case evaluated, the penetration height difference between the circular and slot injections was found to be around $6 \%$. However, slot injection augmentation of laterally affected crossflow increased the side force component coming from crossflow interaction, which finally yielded up to an $8.5 \%$ increase in the global vector side force, as presented in Table 9 .

Table 9 Numerically evaluated forces results ${ }^{\mathrm{a}}$

\begin{tabular}{lcccccccccccc}
\hline & & & & & & \multicolumn{6}{c}{ Performance data } \\
\cline { 7 - 12 } Case & $\dot{m}_{j}, \mathrm{~g} / \mathrm{s}$ & $F_{j y}, \mathrm{~N}$ & $F_{w y}, \mathrm{~N}$ & $\sum F_{y}, \mathrm{~N}$ & $\sum F_{x}, \mathrm{~N}$ & $\delta$, deg & $D_{\mathrm{inj}}, \mathrm{mm}$ & $h_{j}, \mathrm{~mm}$ & $x_{\text {sepj }}, \mathrm{mm}$ & $C_{\mathrm{AV}}$ & $C_{\mathrm{AF}}$ & $C_{\mathrm{AI}}$ \\
\hline Circular & 19.63 & 10.21 & 7.71 & 17.92 & 136.23 & 7.493 & $d_{j}=6$ & 5.512 & 15.664 & 0.914 & 1.025 & 0.946 \\
Slot & 19.83 & 10.23 & 9.43 & 19.66 & 136.64 & 8.187 & $b_{j}=2.5$ & 5.205 & 17.595 & 0.986 & 1.029 & 0.951 \\
\hline
\end{tabular}




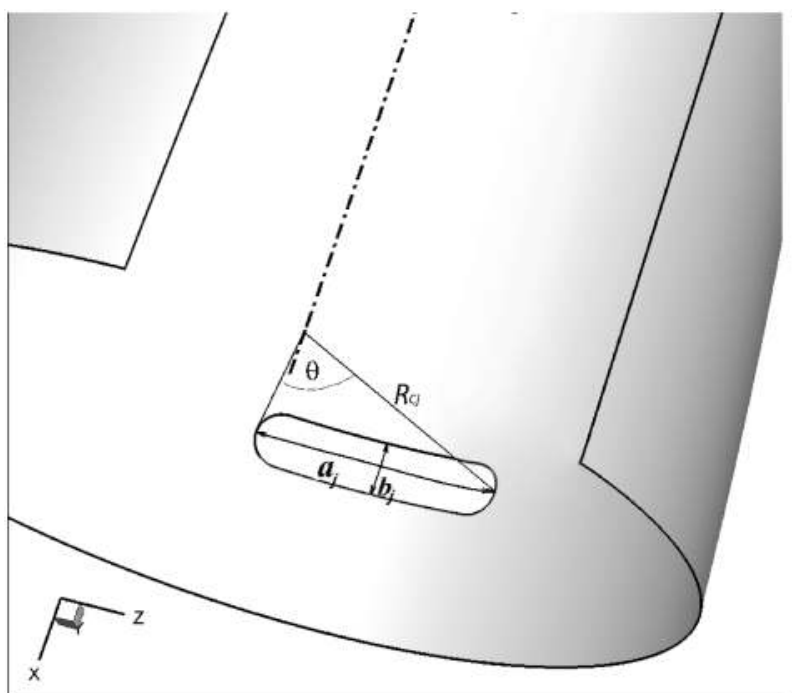

a) Slot central arc definition

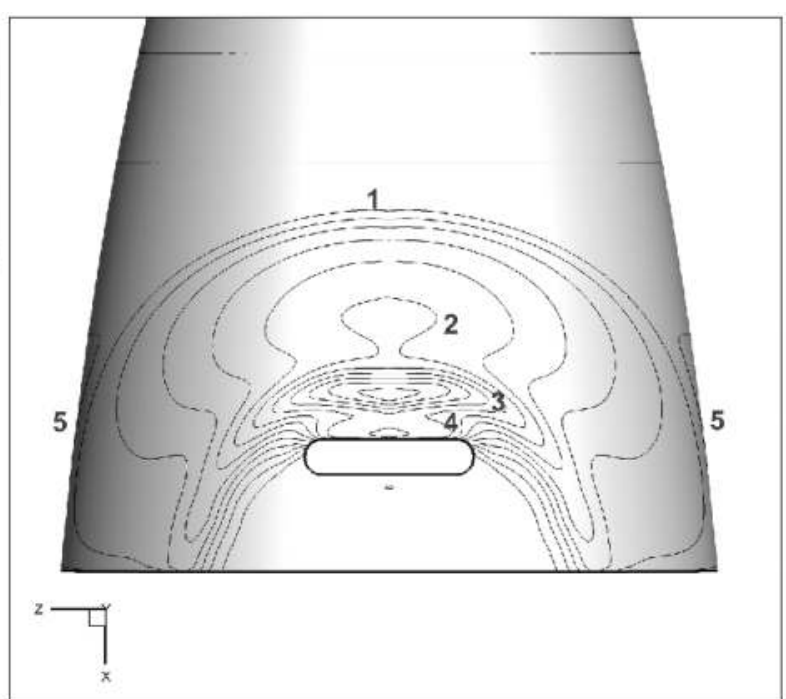

b) Numerically evaluated pressure contours

Fig. 31 Slot injection scheme at $\mathrm{SPR}=1$ and $\mathrm{NPR}=37.5$ conditions.

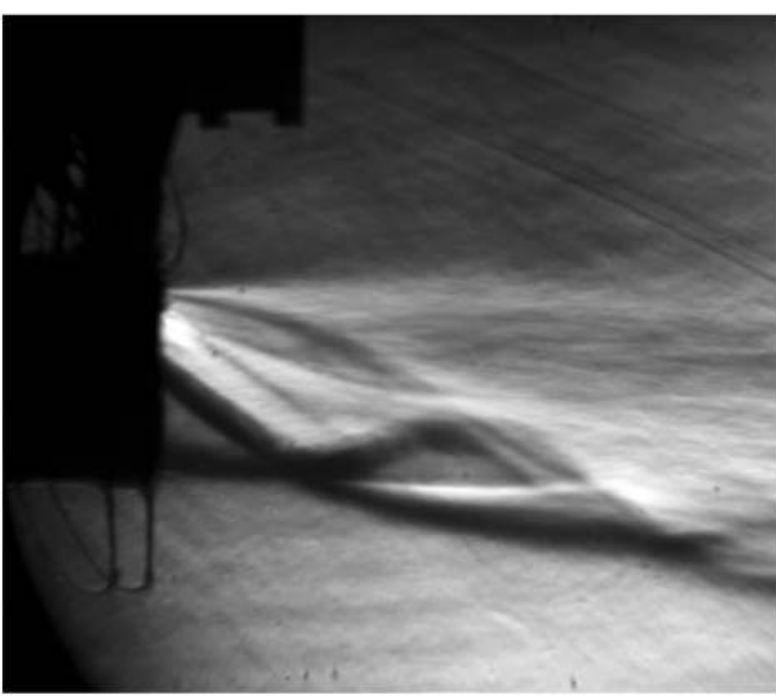

a) Side-view experimental schlieren

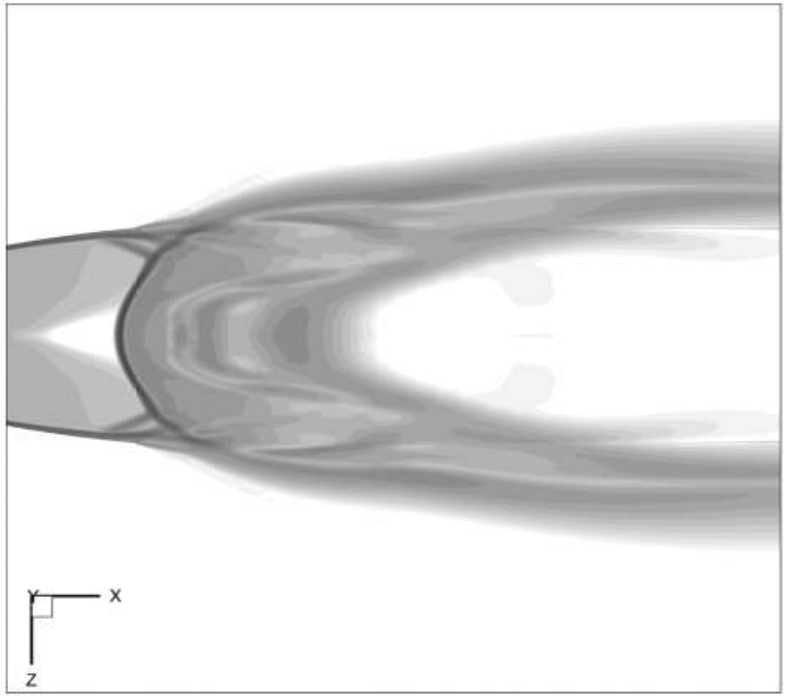

b) Top-view numerical schieren

Fig. $32 Z$ schlieren for slot injection case at $S P R=1$ and NPR $=37.5$ conditions, yielding $\delta=8.18$ deg.

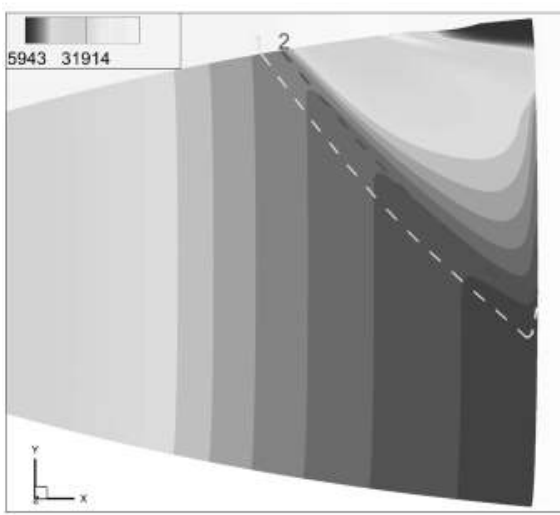

a) Separation zone displacement

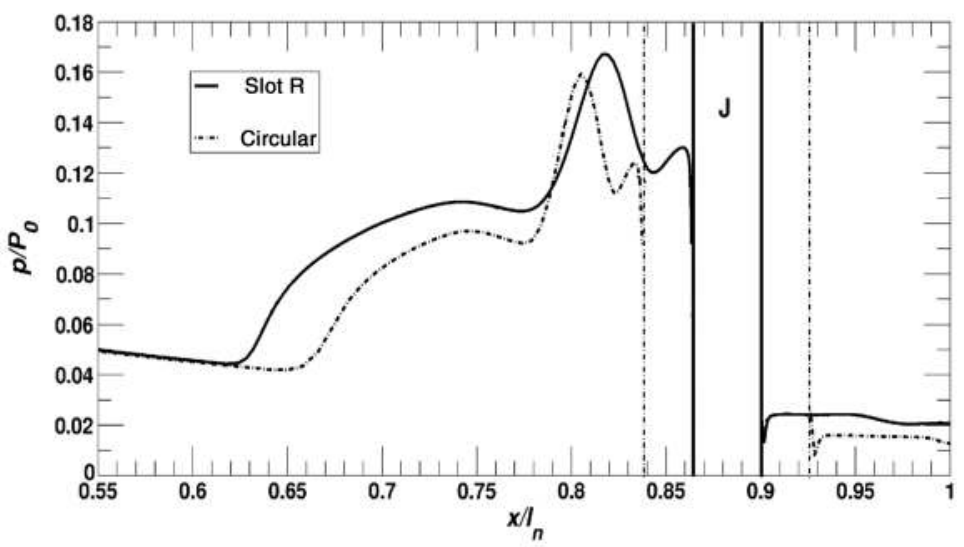

b) Wall-pressure profiles for circular and slot injection

Fig. 33 Circular and slot injection case profiles at $S P R=1$ and $N P R=37.5$ conditions. 


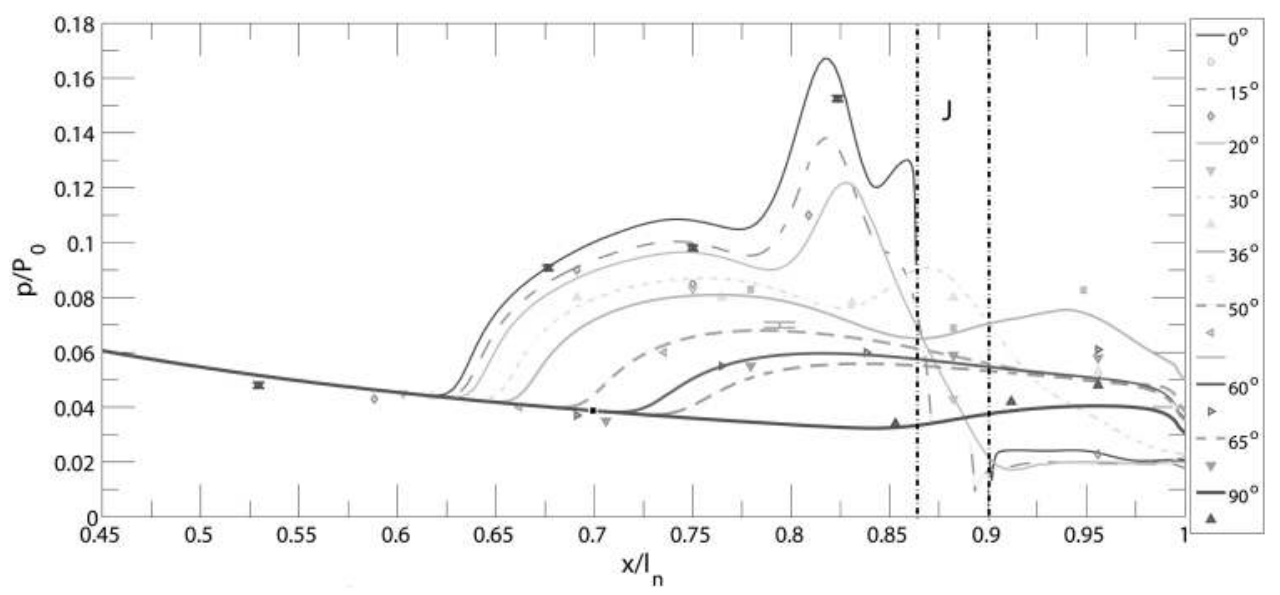

Fig. 34 Wall-pressure at meridional positions for $S P R=1, N P R=37.5$ (lines - CFD, symbols - experiment).

The effects of the slot injection were comparable to the parallel multiport injection distributed along the 30 deg central arc region. This radial kind of multiport injection positioning, as reported in $[33,44]$, yielded a better performance rate than the meridional positioning, and its generated side force was found in the range of a singular circular injection. Some further viable optimizations of geometry aspects were suggested, such as the Rao adverse-pressuregradient optimized nozzle contour $[33,37]$.

\section{Conclusions}

The presented study aimed to address the problematic and possible application approaches of secondary injection fluidic thrust vectoring. The number of identified aspects was parametrically analyzed and presented qualitatively, as well quantitatively. The integrated experimental and numerical approach proved well suited for the posed problem. Very good agreement between the experimental results and computational fluid dynamics with the $k-\varepsilon$ model was reached in terms of the estimation of the separation zone and the plateau pressure rise.

The main features of the complex wall-bounded interaction crossflow field were analyzed. In addition to adverse-pressuregradient shock generation and interaction with a secondary plume, cold-flow intrinsic gas properties were considered. In Mach 3 compressible flow, it was detected that the pressure condition, and thus the sonic velocity of the gas, mainly influenced the penetration rate, implicit separation, and effective main flow deflection. Therefore, species with a lower $\gamma$ molar mass ratio proved as more efficient compared to inert gases.

A number of geometric parameters was tested for the given rocket nozzle model. Injection position and injection inclination toward the main nozzle axis proved to dominantly affect the secondary injection thrust vector control (SITVC) performances. Considering the shock reflection and interaction inside the nozzle, as well as the imbalance of the high-pressure upstream zone and low pressure downstream of injection, the positions closer to the nozzle exit proved a better selection for the SITVC implementation. Upstream inclination ameliorated the amount of side force coming from crossflow interaction. However, at high injection inclination angles, the negative effects of backpressure action on the injection port and instable shear flow close to the wall globally deteriorated nozzle operation. Optimization of secondary nozzle geometry proved to have a moderate effect on the performances. Slot injection on the 30 deg central arc affected a larger amount of the main flow laterally, which increased the interaction side force component.

With directions obtained from the presented cold-flow investigation, a hot-gas analysis of the combustion chamber products in the SITVC nozzle is currently under investigation. Additionally, the effects of flow instability modes at the SITVC nozzle exit will be considered in the ongoing investigation.

\section{Acknowledgments}

The authors would like to acknowledge the full technical and financial support of the Centre National d'Etudes Spatiales (CNES) French space agency: particularly, the great cooperation with Sandrine Palerm and Jean Oswald of CNES/Direction des Lanceurs. Also, we greatly appreciate the technical assistance of Viviana Lago and Nicolas Gouilon during all of the conducted experiments.

\section{References}

[1] Hingre, H., Bec, R., Palerm, S., and Oswald, J., "European University and Scientific Space Research Program PERSEUS," EUCASS 4th European Conference for AeroSpace Sciences, Russian Foundation for Basic Research, S-8.8, St. Petersburg, Russia, 2011.

[2] Flamm, J. D., "Experimental Study of a Nozzle Using Fluidic Counterflow for Thrust Vectoring," 34th AIAA/ASME/SAE/ASEE Joint Propulsion Conference and Exhibit, AIAA Paper 1998-3255, 1998. doi:10.2514/6.1998-3255

[3] Sung, H.-G., and Heo, J.-Y., "Fluidic Thrust Vector Control of Supersonic Jet Using Coflow Injection," Journal of Propulsion and Power, Vol. 28, No. 4, 2012, pp. 858-861. doi:10.2514/1.B34266

[4] Deere, K. A., "Summary of Fluidic Thrust Vectoring Research at NASA Langley Research Center," 21st AIAA Applied Aerodynamics Conference, AIAA Paper 2003-3800, 2003. doi: $10.2514 / 6.2003-3800$

[5] Mahesh, K., "The Interaction of Jets with Crossflow," Annual Review of Fluid Mechanics, Vol. 45, Jan. 2013, pp. 379-407. doi:10.1146/annurev-fluid-120710-101115

[6] Viti, V., Neel, R., and Schetz, J. A., "Detailed Flow Physics of the Supersonic Jet Interaction Flow Field," Physics of Fluids, Vol. 21, No. 4, 2009, Paper 046101 doi:10.1063/1.3112736

[7] Zukoski, E. E., and Spaid, F. W., "Secondary Injection of Gases into a Supersonic Flow," AIAA Journal, Vol. 2, No. 10, 1964, pp. 1689-1696. doi: $10.2514 / 3.2653$

[8] Spaid, F. W., and Zukoski, E. E., "Study of the Interaction of Gaseous Jets from Transverse Slots with Supersonic External Flows," AIAA Journal, Vol. 6, No. 2, 1968, pp. 205-212. doi: $10.2514 / 3.4479$

[9] Guhse, R. D., and Doyle Thompson, H., "Some Aspects of Gaseous Secondary Injection with Application to Thrust Vector Control," Journal of Spacecraft and Rockets, Vol. 9, No. 5, 1972, pp. 291-292. doi:10.2514/3.61674

[10] Guhse, R. D., "On Secondary Gas Injections into Supersonic Nozzles," AIAA Journal, Vol. 3, No. 1, 1966, pp. 147-149. doi: $10.2514 / 3.28405$

[11] Schetz, J. A., Billig, F. S., and Favin, S., "Analysis of Slot Injection in Hypersonic Flow," Journal of Propulsion and Power, Vol. 7, No. 1, 1991, pp. 115-122. doi: $10.2514 / 3.2330$

[12] Karamcheti, K., and Tao-Sze Hsia, H., "Integral Approach to an Approximate Analysis of Thrust Vector Control by Secondary Injection," AIAA Journal, Vol. 1, No. 11, 1963, pp. 2538-2544. doi:10.2514/3.2106 
[13] Walker, R. E., and Shandor, M., "Influence of Injectant Properties for Fluid Injection Thrust Vector Control," AIAA Solid Propellant Rocket Conference, AIAA Paper 1964-0112, 1964. doi:10.2514/6.1964-112

[14] Allegre, J., and Charwat, A. F., "Interaction of a Supersonic Stream and a Transverse Supersonic Jet," AIAA Journal, Vol. 2, No. 11, 1964, pp. $1965-1972$. doi: $10.2514 / 3.2712$

[15] Zukoski, E. E., "Turbulent Boundary-Layer Separation in Front of a Forward-Facing Step," AIAA Journal, Vol. 5, No. 10, 1967, pp. 17461753. doi: $10.2514 / 3.4299$

[16] Verma, S. B., and Gupta, V., "Supersonic Separation with Obstructions," AIAA Journal, Vol. 34, No. 4, 1996, pp. 849-851. doi: $10.2514 / 3.13151$

[17] Cuppoletti, D. R., and Gutmark, E., "Fluidic Injection on a Supersonic Jet at Various Mach Numbers," AIAA Journal, Vol. 52, No. 2, 2014, pp. 293-306. doi:10.2514/1.J010000

[18] Santiago, J. G., and Dutton, J., "Crossflow Vortices of a Jet Injected into a Supersonic Crossflow," AIAA Journal, Vol. 35, No. 5, 1997, pp. 915917. doi: $10.2514 / 2.7468$

[19] Haven, B. A., and Kurosaka, M., "Kidney and Anti-Kidney Vortices in Crossflow Jets," Journal of Fluid Mechanics, Vol. 352, Dec. 1997, pp. 27-64. doi:10.1017/S0022112097007271

[20] Newton, J. F., Jr., and Spaid, F. W., "Interaction of Secondary Injectants and Rocket Exhaust for Thrust Vector Control," ARS Journal, Vol. 32, No. 8, 1962, pp. 1203-1211. doi: $10.2514 / 8.6245$

[21] Ko, H., and Yoon, W.-S., "Performance Analysis of Secondary Gas Injection into a Conical Rocket Nozzle," Journal of Propulsion and Power, Vol. 18, No. 3, 2002, pp. 585-591. doi: $10.2514 / 2.5972$

[22] Zeierman, I., and Manheimer-Timnat, Y., "Full Control of Solid Propellant Rockets by Secondary Injection," Journal of Spacecraft and Rockets, Vol. 10, No. 3, 1973, pp. 161-162 doi: $10.2514 / 3.61863$

[23] Nielson, J. H., Gilchrist, A., and Lee, C. K., "Side Thrust Control by Secondary Gas Injection into Rocket Nozzles," Journal of Mechanical Engineering Science, Vol. 10, No. 3, 1968, pp. 239-251. doi:10.1243/JMES_JOUR_1968_010_036_02

[24] Wing, D. J., and Giuliano, V. J., "Fluidic Thrust Vectoring of an Axisymmetric Exhaust Nozzle at Static Conditions," ASME Fluids Engineering Division Summer Meeting, American Soc. of Mechanical Engineers Paper FEDSM97-3228, Fairfield, NJ, 1997.

[25] Zmijanovic, V., Lago, V., Sellam, M., and Chpoun, A., "Thrust Shock Vector Control of an Axisymmetric Conical Supersonic Nozzle via Secondary Transverse Gas Injection," Shock Waves, Vol. 24, No. 1, 2014, pp. 97-111. doi:10.1007/s00193-013-0479-y

[26] Sellam, M., Zmijanovic, V., Lago, V., and Chpoun, A., "Fluidic Thrust Vectoring of an Axisymmetrical Nozzle: An Analytical Model," International Journal of Aerodynamics, Vol. 2, Nos. 2-4, 2012, pp. 193-209. doi:10.1504/IJAD.2012.049112

[27] Schilling, T. W., "Flow Separation in Rocket Nozzles," M.S. Thesis, Univ. of Buffalo, Buffalo, NY, 1962.

[28] Green, L., Jr., "Flow Separation in Rocket Nozzles," Journal of the American Rocket Society, Vol. 23, No. 1, 1953, pp. 34-35. doi: $10.2514 / 8.4536$

[29] Billig, F. S., "Shock-Wave Shapes Around Spherical-And CylindricalNosed Bodies," Journal of Spacecraft and Rockets, Vol. 4, No. 6, 1967, pp. $822-823$.

doi:10.2514/3.28969
[30] Grunnet, J. L., "Factors Affecting the Magnitude of Jet Interaction Forces," Journal of Spacecraft and Rockets, Vol. 8, No. 12, 1971, pp. 1234-1235 doi: $10.2514 / 3.30370$

[31] Azevedo, D., "Measured Thrust Losses Associated with Secondary Air Injection Through Nozzle Walls," Journal of Propulsion and Power, Vol. 9, No. 1, 1993, pp. 43-50. doi: $10.2514 / 3.11483$

[32] Riebe, J., "Investigation of Gaseous Secondary Injection Thrust Vector Control Considering Primary Nozzle Overexpansion, Frequency Response, and Injection Angle," NASA CR-66533, 1968.

[33] Zmijanovic, V., "Secondary Injection Fluidic Thrust Vectoring of an Axisymmetric Supersonic Nozzle," Ph.D. Dissertation, Doctoral School of Science and Technology, Orleans Univ., Orleans, France, 2013.

[34] Damljanovic, D., Isakovic, J., and Rasuo, B., "T-38 Wind-Tunnel Data Quality Assurance Based on Testing of a Standard Model," Journal of Aircraft, Vol. 50, No. 4, 2013, pp. 1141-1149. doi:10.2514/1.C032081

[35] Zucrow, M. J., and Hoffman, J. D., Gas Dynamics, Vols. 1-2, Wiley, New York, 1977.

[36] Rao, G. V. R., "Approximation of Optimum Thrust Nozzle Contour," ARS Journal, Vol. 30, No. 6, 1960, p. 561.

[37] Rao, G. V. R., "Thrust Vector Control System Utilizing an Adverse Pressure Gradient in the Nozzle," NASA SN/60 NASw-249, 1961.

[38] Durand, P., Vieille, B., Lambaré, H., Vuillermoz, P., Bouré, G., Steinfeld, P., Godfroy, F., and Guéry, J. F., "CPS" "AThree Dimensional CFD Code Devoted to Space Propulsive Flows," 36th AIAA/ASME/SAE/ ASEE Joint Propulsion Conference and Exhibit, AIAA Paper 20003864,2000 doi: $10.2514 / 6.2000-3864$

[39] Toro, E. F., Spruce, M., and Speares, W., "Restoration of the Contact Surface in the HLL-Riemann Solver," Shock Waves, Vol. 4, No. 1, 1994, pp. 25-34. doi:10.1007/BF01414629

[40] Chenault, C. F., and Beran, P. S., "K-e and Reynolds Stress Turbulence Model Comparisons for Two-Dimensional Injection Flows," AIAA Journal, Vol. 36, No. 8, 1998, pp. 1401-1412. doi: $10.2514 / 2.561$

[41] Boccaletto, L., and Cahuzac, F., "Solving the Flow Separation Issue: A New Nozzle Concept," 44th AIAA/ASME/SAE/ASEE Joint Propulsion Conference and Exhibit, AIAA Paper 2008-5234, 2008. doi:10.2514/6.2008-5234

[42] Zmijanovic, V., Leger, L., Lago, V., Sellam, M., and Chpoun, A., "Experimental and Numerical Study of Thrust-Vectoring Effects by Transverse Gas Injection into a Propulsive Axisymmetric C-D Nozzle," 48th AIAA/ASME/SAE/ASEE Joint Propulsion Conference and Exhibit, AIAA Paper 2012-3874, 2012. doi: $10.2514 / 6.2012-3874$

[43] Erinc, E., and Kontis, K., "Numerical and Experimental Investigation of Transverse Injection Flows," Shock Waves, Vol. 20, No. 2, 2010, pp. 103-118. doi:10.1007/s00193-010-0247-1

[44] Waithe, K., and Deere, K. A., "An Experimental and Computational Investigation of Multiple Injection Ports in a Convergent-divergent Nozzle for Fluidic Thrust Vectoring," 21 st AIAA Applied Aerodynamics Conference, AIAA Paper 2003-3802, 2003. doi:10.2514/6.2003-3802

[45] Zeamer, R., "Thrust Vector Control by Liquid Injection for Solid Propellant Rockets," 11th AIAA/SAE Propulsion Conference, AIAA Paper 1975-1225, 1975. doi:10.2514/6.1975-1225

[46] Nielson, J. H., Gilchrist, A., and Lee, C. K., "Control Forces in Rocket Nozzles Produced by a Secondary Gas Stream Inclined at Various Angles to the Nozzle Axis," Journal of Mechanical Engineering Science, Vol. 11, No. 2, 1969, pp. 175-180. doi:10.1243/JMES_JOUR_1969_011_022_02 